\title{
Comparative Genome-wide Analysis and Expression Profiling of Histone Acetyltransferases and Histone Deacetylases Involved in the Response to Drought in Wheat
}

Hua Li ( $\square$ lihuahnnd@163.com )

Henan Agricultural University

Huajie Liu

Henan Agricultural University

Xinxin Pei

Henan Agricultural University

Hongyu Chen

Henan Agricultural University

Xiao Li

Henan Agricultural University

Jiarui Wang

Henan Agricultural University

Chenyang Wang

Henan Agricultural University

\section{Research Article}

Keywords: histone acetyltransferases, histone deacetylases, gene expression, drought stress, wheat

Posted Date: December 15th, 2020

DOI: https://doi.org/10.21203/rs.3.rs-123885/v1

License: (c) (1) This work is licensed under a Creative Commons Attribution 4.0 International License.

Read Full License

Version of Record: A version of this preprint was published at Journal of Plant Growth Regulation on April 13th, 2021. See the published version at https://doi.org/10.1007/s00344-021-10364-9. 


\section{Abstract}

Background: Histone acetyltransferases (HATs) and histone deacetylases (HDACs) contribute to plant growth, development, and stress responses. A number of HAT and HDAC genes have been identified in several plants. However, wheat HATs and HDACs have not been comprehensively characterized. In this study, we identified TaHATs and TaHDACs in the wheat genome using bioinformatics tools.

Result: In total, 30 TaHAT genes and 53 TaHDAC genes were detected in the wheat genome. As described in other plants, TaHATs were classified into four subfamilies (i.e., GNAT, p300/CBP, MYST, and TAFII250) and TaHDACs were divided into three subfamilies (i.e., RPD3/HDA1, HD2, and SIR2). Phylogenetic and conserved domain analyses showed that TaHATs and TaHDACs are highly similar to those in Arabidopsis and rice; however, divergence and expansion from Arabidopsis and rice were also observed. We detected many stress-related cis-regulatory elements in the promoter regions of these genes (i.e., ABRE, STRE, MYB et al.). Further, based on a comparative expression analyses of three varieties with different degrees of drought resistance under drought stress, we found that TaHAG2, TaHAG3, TaHAC2, TaHDA18, TaHDT1, and TaHDT2 are likely regulate drought stress in wheat.

Conclusions: In this study, TaHATs and TaHDACs from the wheat genome were identified. Three TaHATs and three TaHDACs were very likely to regulate drought stress based on a promoter analysis and gene expression analysis. These results provide a foundation for further research on the regulation of acetylation in wheat and its role in the response to drought stress.

\section{Background}

Plant growth, development, and stress responses depend on precise and accurate gene regulatory systems. Epigenetic modification is an important regulatory mechanism underlying gene expression and includes the regulation of transcription via DNA methylation or histone modifications, without changing the DNA sequence. Histone modifications mainly occur on lysine, arginine, and serine residues at the $\mathrm{N}$ terminus of histone $\mathrm{H} 3$ or $\mathrm{H} 4$; the modification types include methylation, acetylation, phosphorylation, ubiquitination, and glycosylation [1]. Among these, acetylation is one kind of well-characterized histone post-translational modification. Histone acetylation mainly occurs on the lysine residues at the $\mathrm{N}$ terminus of histones. It is completed by two dynamic and reversible processes, histone acetylation and deacetylation [2]. Histone acetylation can weaken the interaction between histones and negatively charged DNA, change the surface structure of nucleosomes, and stretch chromatin [3-5], which is conducive to the formation of binding sites for transcriptional regulators. Therefore, histone acetylation tends to induce gene activation. Conversely, histone deacetylation often causes chromatin condensation and inhibits gene transcription [6].

The processes of histone acetylation and deacetylation are catalyzed by histone acetyltransferases (HATs) and histone deacetylases (HDACs), respectively. HATs and HDACs regulate the dynamic balance of histone acetylation levels. In plants, HATs are classified into four distinct subfamilies according to their 
structures: GNAT (GCN5-related N-terminal acetyltransferase), p300/CBP (CREB-binding protein), MYST (MOZ, Ybf2/Sas3, Sas2, and Tip60), and TAFII250 (TATA binding protein-associated factors) [7]. HDACs can be divided into three subfamilies: RPD3/HDA1 (Reduced Potassium Dependency 3/Histone DeAcetylase 1), SIR2 (Silent Information Regulator 2), and HD2 (Histone Deacetylase 2) [8]. The RPD3/HDA1 and SIR2 subfamilies are homologous to yeast HDACs and the HD2 subfamily is plantspecific. Accumulating studies have shown that both HATs and HDACs are involved in the regulation of plant growth and development as well as the response to stress [9-12]. For example, Arabidopsis GCN5 mutants lose their apical advantage and show abnormal anther development [13]. In addition, GCN5 mutations or downregulation increase plant sensitivity to salt, drought, heat, and disease stresses [1416]. The MYST family members $H A M 1$ and $H A M 2$ are involved in the regulation of flowering time, and their inhibition leads to early flowering in Arabidopsis [17]. The histone deacetylase HDA9 prevents seedling traits and germination [18] and negatively regulates salt and drought stress responsiveness in Arabidopsis [19]. HDA19 is involved in drought, heat, and salt responses [20-23]. The overexpression of HDA705 in rice decreases ABA and salt stress resistance during seed germination and enhances osmotic stress resistance during the seedling stage [24].

There is accumulating evidence for the important roles of HATs and HDACs in plant development and responses to environmental stresses. Therefore, to better understand the changes in histone acetylation, it is necessary to comprehensive identify and analyze HATs and HDACs in plant genomes. Genome-wide analyses of HATs have been performed in Arabidopsis [7], rice (Oryza sativa) [25], Brachypodium distachyon [26], litchi (Litchi chinensis Sonn) [27], Vitis vinifera [28], Citrus sinensis [29], and cotton (Gossypium raimondi/ arboretum) [30], and HDACs have been identified in Arabidopsis [31], rice [32], soybean [33], Vitis vinifera [28], litchi [27], tomato [34], and cotton [35]. However, little is known about HATs and HDACs in wheat (Triticum aestivum).

In this study, we first identified genes encoding HATs and HDACs in the wheat genome and then comprehensively analyzed these loci with respect to phylogenetic relationships, gene domain and structure, chromosomal location, and cis-regulatory elements in their promoters. Furthermore, the gene expression patterns of HATs and HDACs in different tissues (i.e., leaf, root, spike, and grain) and under drought stress were analyzed. This study could provide a foundation for further research on the regulation of histone acetylation in wheat and closely related monocots.

\section{Results}

\section{Identification of HATs and HDACs protein in wheat}

To identify HATs and HDACs in the genomes of wheat, a systematic blast search was performed using Arabidopsis and rice sequences as queries. Pfam and InterProScan databases were used to further verify the candidate HATs and HDACs based on structural domains. In total, 30 HATs and 53 HDACs were identified in the wheat genome (Table 1, Table S1). The polypeptide lengths of HATs and HDACs were 438-1796 and 309-693 amino acids, respectively, the predicted molecular weights were 50.14-201.47 
and 33.16-74.54 kDa, and the theoretical isoelectronic point ( $\mathrm{pl}$ ) values were 4.71-8.9 and 4.6-9.42. In addition, the intron-exon organization of these HATs and HDACs were analyzed. The numbers of conserved coding regions ranged from 9 to 21 in HATs and from 1 to 17 in HDACs. With respect to subcellular localization, most HATs were detected in the nucleus, but HDACs were located in the nucleus as well as in the chloroplasts, cytoplasm, mitochondria, cytoskeleton, etc. 
Table 1

Histone acetyltransferases (HATs) and Histone deacetylases (HDACs) identified in wheat Subfamily Gene name Gene ID Protein length Localization HATs family

\begin{tabular}{|c|c|c|c|c|}
\hline \multirow[t]{9}{*}{ GNAT } & TaHAG1A & TraesCS1A02G138200.2 & 507 & nucl, chlo \\
\hline & TaHAG1D & TraesCS1D02G134200.1 & 507 & nucl, chlo \\
\hline & TaHAG1U & TraesCSU02G003200.1 & 507 & nucl, chlo \\
\hline & TaHAG2A & TraesCS5A02G197700.1 & 463 & cyto \\
\hline & TaHAG2B & TraesCS5B02G186000.1 & 463 & cyto \\
\hline & TaHAG2D & TraesCS5D02G193200.1 & 463 & cyto \\
\hline & TaHAG3A & TraesCS2A02G320900.1 & 569 & cyto \\
\hline & TaHAG3B & TraesCS2B02G361800.1 & 569 & cyto \\
\hline & TaHAG3D & TraesCS2D02G341600.1 & 569 & cyto \\
\hline \multirow[t]{3}{*}{ MYST } & TaHAG4A & TraesCS2A02G159700.1 & 438 & nucl, cyto \\
\hline & TaHAG4B & TraesCS2B02G185300.1 & 482 & nucl, mito \\
\hline & TaHAG4D & TraesCS2D02G166900.1 & 438 & nucl, cyto \\
\hline \multirow[t]{12}{*}{ CBP } & TaHAC1A & TraesCS3A02G524800.1 & 1286 & nucl \\
\hline & TaHAC1B & TraesCS3B02G592100.1 & 1288 & nucl \\
\hline & TaHAC1D & TraesCS3D02G530000.2 & 1286 & nucl \\
\hline & TaHAC2A & TraesCS2A02G039500.3 & 1186 & nucl \\
\hline & TaHAC2B & TraesCS2B02G052300.4 & 1186 & nucl \\
\hline & TaHAC2D & TraesCS2D02G038100.1 & 1185 & nucl \\
\hline & TaHAC4A & TraesCS7A02G414500.1 & 1518 & nucl \\
\hline & TaHAC4B & TraesCS7B02G314400.2 & 1512 & nucl, pero \\
\hline & TaHAC4D & TraesCS7D02G407600.2 & 1518 & nucl, pero \\
\hline & TaHAC5A & TraesCS6A02G107300.1 & 1726 & nucl \\
\hline & TaHAC5B & TraesCS6B02G135800.1 & 1726 & nucl \\
\hline & TaHAC5D & TraesCS6D02G095400.1 & 1728 & nucl \\
\hline \multirow[t]{2}{*}{ TAFII250 } & TaHAF1A & TraesCS7A02G515000.1 & 1782 & chlo \\
\hline & TaHAF1B & TraesCS7B02G431700.2 & 1762 & nucl \\
\hline
\end{tabular}




\begin{tabular}{|c|c|c|c|c|}
\hline Subfamily & Gene name & Gene ID & Protein length & Localization \\
\hline & TaHAF1D & TraesCS7D02G505400.1 & 1762 & nucl \\
\hline & TaHAF2A & TraesCS7A02G514800.1 & 1796 & nucl \\
\hline & TaHAF2B & TraesCS7B02G431500.1 & 1796 & nucl \\
\hline & TaHAF2D & TraesCS7D02G505200.1 & 1796 & nucl \\
\hline \multicolumn{5}{|c|}{ HDACs family } \\
\hline \multirow[t]{23}{*}{ RPD3/HDA1 } & TaHDA2A & TraesCS7A02G362600.1 & 355 & chlo, cyto \\
\hline & TaHDA2B & TraesCS7B02G266000.1 & 353 & chlo \\
\hline & TaHDA2D & TraesCS7D02G360500.1 & 353 & chlo \\
\hline & TaHDA5A & TraesCS1A02G317100.1 & 397 & chlo \\
\hline & TaHDA5B & TraesCS1B02G329500.1 & 390 & cyto, chlo \\
\hline & TaHDA5D & TraesCS1D02G317100.1 & 394 & chlo \\
\hline & TaHDA6A & TraesCS6A02G181100.1 & 458 & cysk \\
\hline & TaHDA6B & TraesCS6B02G210200.1 & 458 & cysk, cyto \\
\hline & TaHDA6D & TraesCS6D02G168400.1 & 458 & cysk \\
\hline & TaHDA7A & TraesCS6A02G184100.2 & 519 & nucl \\
\hline & TaHDA7B & TraesCS6B02G212600.3 & 520 & nucl \\
\hline & TaHDA7D & TraesCS6D02G171000.1 & 574 & chlo, nucl \\
\hline & TaHDA8A & TraesCS1A02G275300.1 & 391 & chlo \\
\hline & TaHDA8B & TraesCS1B02G284500.1 & 393 & chlo \\
\hline & TaHDA8D & TraesCS1D02G274900.1 & 366 & chlo \\
\hline & TaHDA9A & TraesCS2A02G293200.1 & 430 & cyto \\
\hline & TaHDA9B & TraesCS2B02G309700.1 & 430 & cyto \\
\hline & TaHDA9D & TraesCS2D02G291000.1 & 430 & cyto \\
\hline & TaHDA14A & TraesCS5A02G119300.2 & 444 & chlo \\
\hline & TaHDA14B & TraesCS5B02G121300.1 & 453 & chlo \\
\hline & TaHDA14D & TraesCS5D02G126600.1 & 444 & chlo \\
\hline & TaHDA15A & TraesCS5A02G065300.1 & 614 & nucl \\
\hline & TaHDA15B & TraesCS5B02G072100.1 & 614 & nucl \\
\hline
\end{tabular}




\begin{tabular}{|c|c|c|c|c|}
\hline Subfamily & Gene name & Gene ID & Protein length & Localization \\
\hline & TaHDA15D & TraesCS5D02G076100.1 & 612 & nucl \\
\hline & TaHDA18A & TraesCS2A02G177100.1 & 693 & chlo, nucl \\
\hline & TaHDA18B & TraesCS2B02G204100.1 & 693 & chlo, nucl \\
\hline & TaHDA18D & TraesCS2D02G185200.1 & 693 & nucl, chlo \\
\hline & TaHDA19A & TraesCS7A02G365600.3 & 523 & nucl \\
\hline & TaHDA19B & TraesCS7B02G261800.1 & 519 & nucl \\
\hline & TaHDA19D & TraesCS7D02G356800.1 & 519 & nucl \\
\hline & TaHDA20A & TraesCS4A02G213200.1 & 471 & mito, nucl \\
\hline & TaHDA20B & TraesCS4B02G102600.1 & 471 & mito, nucl \\
\hline & TaHDA20D & TraesCS4D02G100000.1 & 471 & cyto, nucl \\
\hline & TaHDA21A & TraesCS5A02G295000.1 & 484 & cyto \\
\hline & TaHDA21D & TraesCS5D02G302400.1 & 495 & cyto \\
\hline & TaHDA22B & TraesCS3B02G318000.1 & 380 & cysk \\
\hline & TaHDA22D & TraesCS3D02G422300.1 & 327 & cyto \\
\hline \multirow[t]{10}{*}{ HD2 } & TaHDT1A & TraesCS1A02G445700.4 & 309 & nucl \\
\hline & TaHDT1D & TraesCS1D02G454400.2 & 311 & nucl \\
\hline & TaHDT2A & TraesCS3A02G415200.1 & 403 & nucl \\
\hline & TaHDT2B & TraesCS3B02G450300.1 & 383 & nucl \\
\hline & TaHDT2D & TraesCS3D02G410300.2 & 364 & nucl \\
\hline & TaHDT3B & TraesCS3B02G450400.1 & 378 & nucl \\
\hline & TaHDT3D & TraesCS3D02G410400.1 & 432 & nucl \\
\hline & TaHDT4A & TraesCS5A02G158900.1 & 432 & nucl \\
\hline & TaHDT4B & TraesCS5B02G156700.1 & 433 & nucl \\
\hline & TaHDT4D & TraesCS5D02G164000.1 & 433 & nucl \\
\hline \multirow[t]{4}{*}{ SIR2 } & TaSRT1A & TraesCS2A02G077800.1 & 440 & cyto, nucl \\
\hline & TaSRT1B & TraesCS2B02G092700.1 & 465 & cyto, nucl \\
\hline & TaSRT1D & TraesCS2D02G075800.1 & 678 & nucl, E.R \\
\hline & TaSRT2A & TraesCS5A02G114700.3 & 414 & cyto \\
\hline
\end{tabular}




\begin{tabular}{|lllll|}
\hline Subfamily & Gene name & Gene ID & Protein length & Localization \\
& TaSRT2D & TraesCS5D02G124700.1 & 396 & chlo \\
\cline { 2 - 5 } & TaSRT2U & TraesCSU02G136000.1 & 396 & chlo \\
\hline
\end{tabular}

\section{Phylogenetic and conserved domain analyses of HATs and HDACs in wheat}

To reveal the evolutionary relationships among HATs and HDACs in wheat, a phylogenetic tree was constructed using MEGA 6.0 based on the amino acid sequences (Table S1) for the newly identified HAT and HDAC proteins in wheat and previously identified HATs from Arabidopsis thaliana and rice. Similar to Arabidopsis and rice, wheat HATs could be grouped into four distinct subfamilies: 12 HATs belonged to the CBP subfamily, 9 HATs belonged to the GNAT subfamily, 3 HATs belonged to the MYST subfamily, and 6 HATs belonged to the TAFII250 subfamily (Fig. 1, Table 1). The 53 HDACs in wheat could be classified into three subfamilies, RPD3/HDA1, HD2, and SIR2, with 37, 10, and 6 loci, respectively (Fig. 2, Table 1). HATs and HDACs in wheat were named based on the nomenclature suggestions for Arabidopsis; each gene was assigned a two-letter code corresponding to $T$. aestivum $(\mathrm{Ta})$, followed by family designation and number, followed by $A, B$, or $D$ (according to the subgenome in wheat).

In an analysis of domain architectures, all TaHAT subfamilies in wheat had conserved domains; for example, the CBP subfamily of wheat TaHATs contained the HAT-KAT11 domain, the GNAT subfamily of TaHATs contained the Hat1_N or Acetyltransferase domain, the MYST subfamily contained MOZ_SAS, zfMYST, and Tudor-knot domains, and the TAFII250 subfamily contained DUF3591 and Bromodomain. In addition to these highly conserved domains, the CBP subfamily also contained the PHD, ZZ, and zf-TAZ domains, the GNAT subfamily had the Radical_SAM domain and Bromodomain, and the TBP-binding and ubiquitin domain was found in the TAFII250 subfamily (Fig. 1). For HDACs of wheat, RPD3/HDA1, HD2, and SIR2 subfamilies had the conserved domains Hist-deacetyl, NPL, and SIR2, respectively. In addition, TaHDT2 and TaHDT4 in the HD2 subfamily contained zf-C2H2_6 and FKBP_C domains, respectively. TaSRT2A in SIR2 subfamilies contained the Fibrillarin domain (Fig. 2). In general, wheat HATs and HDACs had similar domain organizations to those of their counterparts in Arabidopsis and rice.

\section{Genomic localization of TaHATs and TaHDACs}

The newly identified wheat HATs and HDACs were mapped to chromosomes. Both TaHATs and TaHDACs were unevenly distributed along the chromosomes (Fig. 3, Figure S1). In particular, there were no TaHAT genes on chromosomes $4 A / B / D$ and $1 B$, three TaHATs were located on chromosomes $2 A / B / D$ and $7 \mathrm{~A} / \mathrm{B} / \mathrm{D}$, and the remaining chromosomes had only a single TaHAT gene. However, TaHDACs were distributed across all chromosomes, with the greatest number on chromosomes 5A/D (five HDACs) and 


\section{Putative cis-regulatory elements in the promoter regions of TaHATs and TaHDACs}

To gain more insight into the putative functions of TaHATs and TaHDACs, the promoter region (1500 bp upstream of the transcription start site) was scanned using the PlantCARE database. Many putative cisregulatory elements were detected in the promoters of both TaHATs and TaHDACs (Fig. 4; Table S2; Table S3), such as ABRE (abscisic acid-responsive element), STRE (stress-responsive element), ARE (essential for anaerobic induction), CCGTCC-box (meristem-specific activation), G-Box (light responsiveness), MYB (MYB-related binding sites), and TGA-element (auxin-responsive element). Most TaHATs and TaHDACs had ABRE (25 HAT genes and 43 HDAC genes) or STRE (27 HAT genes and 46 HDAC genes) elements. However, only TaHDACs had the GARE-motif (gibberellin-responsive element), indicating that the transmission and regulation of GA may be more closely related to histone deacetylation. Moreover, in TaHATs, the number of ABRE elements was higher in all genes in the GNAT subfamily as well as $T a H A C 4 A / B / D$ and TaHAC5A/B/D in the CBP subfamily than in the TAFII250 subfamily, which contained few or no $A B R E$ elements. Genes with a large number of ABRE elements in $H D A C s$ were $T a H D A 5 A / B / D$, $T a H D A 8 A / B / D$, and TaHDA9B/D in the RPD3/HDA1 subfamily, TaHDT2A/B in the HD2 subfamily, and TaSRT1D and TaSRT2Uin the SIR2 subfamily. These genes may mediate the ABA signaling pathway. The TGA-element was only detected in GNAT subfamily genes, such as TaHAG1A/B/Uand TaHAG2A/B/D, and was not observed in genes of the CBP and MYST subfamilies (except TaHAC1D). In general, the distribution of cis-acting elements was more similar in TaHAT subfamilies than in TaHDACs subfamilies.

\section{Expression analysis of TaHATs and TaHDACs in different tissues}

The RNA-seq data for different tissues were obtained from expVIP [36] (Fig. 5). All TaHATs and TaHDACs were differentially expressed in the leaf, root, spike, and grain. TaHAG4A/B/D was expressed in all four tissues and showed the highest expression levels among TaHATs. TaHAC1A/B/D and TaHAF1B/D had very low or no expression in these four tissues. The expression levels of $T a H A G 2 A / B / D$ in the leaf and $T a H A C 4 B / D, T a H A C 5 A / B$, and TaHAF2A/B in the grain were nearly undetectable (Fig. 5A). TaHDT1D was expressed in all four tissues and showed relatively higher expression levels than those of other TaHDACs, while TaHDA20A/B/D, TaHDA21A/D, TaHDA22D, TaHDT1A, TaHDT2A, and TaHDT3B/D were almost undetectable in the four tissues (Fig. $5 B$ ). Additionally, the expression levels differed among $A, B$, and $D$ genomes, e.g., TaHDA19A was not expressed in the leaf, root, or spike, while TaHDA19B/D were highly expressed. TaHDA22B was highly expressed in all four tissues, unlike TaHDA22D (Fig. 5B). These results suggested that the $A, B$, and $D$ genomes may jointly contribute to their functional roles. 


\section{Expression analysis of TaHATs and TaHDACs in response to drought stress}

The identification of putative cis-regulatory elements in the promoter regions suggested that TaHATs and TaHDACs contribute to the response to abiotic stresses. In the main wheat-producing area, plants often encounter drought, leading to reduced yields. Therefore, we focused on the expression of TaHATs and TaHDACs under drought stress. Drought resistance is significantly higher in the wheat variety BN207 than in its parents BN64 and ZM16 (Table S4). Therefore, we used these varieties to identify TaHATs and TaHDACs that may contribute to the response to drought stress by comparative expression analyses.

Similar to the tissue expression results, TaHDA5, TaHDA20, TaHDA21, and TaHDT3 were not detected in the leaf by qRT-PCR. For the remaining TaHATs and TaHDACs, regardless of conditions (i.e., normal or drought), a number of TaHATs and TaHDACs were significantly differentially expressed among the three varieties. However, the expression levels of TaHAG4 and TaHAF1 in the TaHAT family and TaHDA7, TaHDA15 and TaSRT2 in the TaHDACs family were not affected or were slightly affected by drought stress in all three varieties (Fig. 6). This indicated that these genes may not be related to the regulation of drought stress. All other genes were up-regulated or down-regulated in at least one variety under drought stress. It is worth noting that TaHAG2, TaHAG3, and TaHAC2 in the TaHAT family (Fig. 6A) and TaHDA2, TaHDA18, TaHDT1, and TaHDT2 in the TaHDAC family (Fig. 6B) showed a significant response to drought stress only in BN207; in particular, TaHAG2, TaHAG3, TaHAC2, and TaHDT1 were up-regulated under drought stress, while TaHDA2, TaHDA18, and TaHDT2 were down-regulated. Further, the expression of these genes (except TaHDA2) in BN207 under drought stress was significantly different from levels in its parents BN64 and ZM16. Therefore, combined with the observation that BN207 had higher drought resistance than that of its parents BN64 and ZM16, our results suggested that these six genes were likely to mediate drought stress in wheat.

\section{Discussion}

\section{Characterization of TaHATs and TaHDACs proteins}

HATs and HDACs could mediate plant growth and development and the response to environmental stresses [10]. A number of HATs and HDACs have been identified in several plants. However, little is known about these enzymes in wheat. In this study, 30 TaHATs and 53 TaHDACs were identified in the wheat genome using bioinformatics tools (Table 1, Table S1). Wheat is hexaploid; accordingly, the numbers of TaHATs and TaHDACs are approximately three times those of Arabidopsis and rice [7, 25, 32]. All subfamilies of TaHATs and TaHDACs contained subfamily-specific domains, as in other plants. Further, TaHATs and TaHDACs also contain other conserved domains, such as zf-TAZ, ZZ, PHD, and Bromodomains. The zf-TAZ, ZZ, and PHD domains are thought to be involved in protein recognition and protein-protein interactions [37-38]. Bromodomains bind to acetylated lysine residues [39-40]. In general, the protein domains of wheat TaHATs and TaHDACs are highly similar to those of homologues in 
Arabidopsis and rice, suggesting that all these genes in wheat have similar functions to those described in other plant species.

\section{Evolution and function divergence of TaHATs and TaHDACs}

All HATs (except AtHAG5 and AtHAC12) in Arabidopsis correspond to homologous genes on the A, B, and D genomes of wheat (Fig. 1). Previous studies have also shown that homologues of AtHAG5 and AtHAC12 are lacking in Brachypodium distachyon [26]. Homologous genes of AtHAC2 and AtHAF2 were detected in wheat (Fig. 1) but not in B. distachyon [26]. Similar results were found in the HADC family; for example, ten genes in the HD2 subfamily were found in wheat, corresponding to four HD2 subfamily genes in Arabidopsis (AtHD1, AtHD2, AtHD3, and AtHD4) (Fig. 2), but only two HD2 subfamily genes exist in rice (OsHD701 and OsHD702) [32]. In addition, TaHDA21A/D and TaHDA22B/D in the RPD3/HDA1 subfamily have the conserved Hist-deacetyl domain, but they have low homology with all RPD3/HDA1 subfamily members in Arabidopsis. These results indicated that compared with the dicotyledonous Arabidopsis and the monocotyledonous rice, wheat TaHAT and TaHDAC families have diverged and expanded.

A subcellular localization analysis showed that a majority of TaHATs (77\%) are located in the nucleus. However, only $38 \%$ of TaHDAC family members were located in the nucleus; the others were mainly located in the chloroplast (30\%) and cytoplasm (21\%) (Table 1). These results indicated that TaHATs and TaHDACs may have vital functions other than histone acetylation. In particular, the TaHDAC family may have broader functions than those of the TaHAT family based on the predominant localization in the chloroplast and cytoplasm (Table 1). In fact, lysine-acetylated proteins are located in various cellular compartments in Arabidopsis and rice [41-43]. In rice, $45 \%$ of acetylated proteins are distributed in the chloroplast, followed by the cytoplasm (27\%), and only $13 \%$ of acetylated proteins are located in the nucleus [43]. Similarly, a large number of acetylated proteins are related to photosynthesis in Arabidopsis [41], e.g., photosystem II (PSII) subunits, light-harvesting chlorophyll a/b-binding proteins (LHCb), Rubisco large and small subunits, and chloroplastic ATP synthase (b-subunit).

\section{Expression level of TaHATs and TaHDACs under drought stress}

Numerous studies have confirmed that HATs and HDACs are involved in the regulation of plant abiotic stresses. A number of histone acetylation modification genes that regulate drought resistance have recently been identified in various plants. For instance, the histone acetylase GCN5 in Populus trichocarpa can improve the drought resistance of plants by enhancing H3K9ac and enriching RNA polymerase II at PtrNAC genes [15]. AtHDA9 can regulate the acetylation level in the promoters of stress-responsive genes and thus negatively regulate plant sensitivity to salt and drought stresses in Arabidopsis [19]. The overexpression of the Populus histone deacetylase gene $84 \mathrm{KHDA903}$ in tobacco enhances drought tolerance [44]. The silencing of the tomato histone deacetylase gene SIHDA5 results in reduced tolerance to salt, drought, and ABA [45]. The overexpression of the Brachypodium histone deacetylase BdHD1 results in 
hypersensitivity to $A B A$ and enhanced drought resistance [46]. The overexpression of the cotton histone deacetylase GhHDT4D in Arabidopsis increases plant tolerance to drought [47]. In this study, we analyzed the expression changes of all TaHATs and TaHDACs under drought stress in different drought-resistant wheat varieties. Different TaHATs and TaHDACs had different expression profiles in the three varieties (Fig. 6). However, under drought stress, TaHAG2, TaHAG3, TaHAC2, and TaHDT1 levels were significantly higher in the drought-resistant variety BN207 than in BN64 and ZM16. Conversely, TaHDA18 and TaHDT2 were significantly down-regulated by drought in BN207. BN64 and ZM16 are the parents of BN207; however, BN207 shows higher drought resistance (Table S4), implying that the six genes described above are likely to participate in the regulation of drought stress. Based on a promoter analysis, these six genes contain a large number of STRE or ABRE elements (Fig. 4). TaHAG2, TaHAG3, TaHDA18, and TaHDT2 had multiple $A B A$ elements, suggesting that the regulation of drought stress by these four genes may depend on the ABA signaling pathway. TaHAC2 and TaHDT1 contained no or few ABRE elements, indicating that the responses of these loci to drought may be ABA-independent.

\section{Conclusions}

HATs and HDACs exert important functions related to plant growth, development, and stress responses. In this study, TaHATs and TaHDACs in the wheat genome were identified, and the expression patterns of these genes in various tissues and under drought stress were analyzed. In total, 30 TaHATs and 53 TaHDACs were found in wheat and were classified into four and three subfamilies, respectively. Further, based on expression analyses, we found that TaHAG2, TaHAG3, TaHAC2, TaHDA18, TaHDT1, and TaHDT2 are closely related to drought stress. In summary, this study provides a foundation for further studies of TaHATs and TaHDACs in wheat and their regulatory roles in the response to drought stress.

\section{Methods}

\section{Identification of HATs and HDACs}

Wheat HAT and HDAC proteins were identified following previously described methods in studies of Arabidopsis [7] and rice [25]. The HAT and HDAC protein sequences from Arabidopsis and rice were downloaded from Phytozome (http://phytozome.jgi.doe.gov/pz/portal.html) and used as queries for searches against the T. aestivum $\mathrm{L}$. genomes using BLASTP in Ensembl

(http://plants.ensembl.org/index.html). Furthermore, the predicted HATs and HDACs were confirmed by searching the protein domains in Pfam (http://pfam.xfam.org/search) and InterPro database (http://www.ebi. ac.uk/interpro/search/sequence-search).

The physicochemical properties of predicted HAT and HDAC proteins were predicted using ExPASy (http://cn.expasy.org/tools). The subcellular localization of each HAT and HDAC protein was predicted using WoLF PSORT (https://www.genscript.com/wolf-psort.html). 


\section{Phylogenetic and domain analysis}

To investigate evolutionary relationships, a multiple sequence alignment was generated for HATs and HDACs proteins in Triticum aestivum, Oryza sativa, and Arabidopsis thaliana using ClustalW with default parameters. An un-rooted phylogenetic tree was constructed based on the neighbor-joining method with 1000 bootstrap replicates using MEGA6 [48]. Domain annotation was performed using Pfam, and TBtools was used for visualization [49].

\section{Chromosomal distribution and localization}

The positional information of TaHATs and TaHDACs was downloaded from Ensembl database, and then their chromosomal distribution was mapped using TBtools [49].

\section{Promoter cis-element analysis}

The $1.5 \mathrm{~kb}$ upstream sequences of the transcription start site were downloaded from IWGSC (http://www.wheatgenome.org/), and then the PlantCARE database (http://bioinformatics.psb.ugent.be/webtools/plantcare/html/) was used to analyze cis-regulatory elements.

\section{Plant materials and stress treatments}

The wheat cultivar BN207 and its parents BN64 and ZM16 plants were used for gene expression analyses. Seeds were sown into $27 \times 26 \mathrm{~cm}$ pots filled with cultivated soil, with 12 plants per pot. Growth conditions and management were the same as those for field cultivation. Drought treatment was performed at the booting stage of wheat, using $75 \%$ soil water as a control and $55 \%$ soil water as a drought treatment. Flag leaves were collected for a gene expression analysis after 10 days of drought. After 15 days of treatment, plants were rehydrated and the yield was measured at the mature period.

\section{Tissue expression analysis}

The expression levels (TPM values) of TaHATs and TaHDACs in the leaf, root, spike, and grain were downloaded from expVIP (http://www.wheat-expression.com/) [36]. A heatmap was generated using Heml (http://hemi.biocuckoo.org/down.php).

\section{Quantitative real-time PCR analysis}


Total RNA was isolated using the Omniplant RNA Kit (CWBIO, Beijing, China) following the manufacture's protocols. Then, $2 \mu \mathrm{g}$ of total RNA was used to synthesize cDNA with cDNA Synthesis Supermix (Vazyme, Nanjing, China). qRT-PCR was performed using AceQ ${ }^{\text {TM }}$ Universal SYBR qPCR Master Mix (Vazyme) and the ABI StepOnePlus Real-Time PCR System. The expression levels were calculated using the $2^{-\Delta C t}$ method. TaACT-1 was used as a housekeeping gene for normalization [50]. The primers used in this study are listed in Table 55.

\section{Statistical analysis}

The qRT-PCR results are reported as the means of three independent experiments. Differences among treatments were analyzed by one-way ANOVA and Duncan's multiple range tests, setting $p<0.05$ as the threshold for significance.

\section{Declarations}

\section{Acknowledgments:}

Not applicable.

\section{Author Contributions:}

$\mathrm{HL}$ designed the experiments and wrote the manuscript. $\mathrm{HL}$ and $\mathrm{HJL}$ carried out the bioinformatics analysis. XXP and HYC performed the biological experiment. XL and JRW prepared the plant sample. $C Y W$ helped to revise the manuscript. All authors read and approved the final manuscript.

\section{Funding:}

This research was supported by the National Key Research and Development Program of China (2017YFD0300408).

\section{Availability of data and materials:}

All data generated or analyzed during this study are included in this published article and its Additional files. The datasets generated and analyzed during the current study are available from the corresponding author on reasonable request.

\section{Ethics approval and consent to participate:}

Not applicable. 


\section{Consent for publication:}

Not applicable.

\section{Competing interests:}

The authors declare that they have no competing interests.

\section{References}

1. Kouzarides T. Chromatin modifications and their function. Cell. 2007; 128:693-705.

2. Hassig CA, Schreiber SL. Nuclear histone acetylases and deacetylases and transcriptional regulation: HATs off to HDACs. Curr Opin Chem Biol. 1997; 1:300-308.

3. Hollender C, Liu Z. Histone deacetylase genes in ArabidopsisJ Integr Plant Biol. 2008; 50:875-885.

4. Allis CD, Jenuwein T. The molecular hallmarks of epigenetic control. Nat Rev Genet. 2016;17:487500.

5. Onufriev AV, Schiessel $H$. The nucleosome: from structure to function through physics. Curr Opin Struc Biol. 2019; 56:119-130.

6. Shahbazian M, Grunstein M. Functions of site-specific histone acetylation and deacetylation. Annu Rev Biochem. 2007; 76:75-100.

7. Pandey R, MuĖller A, Napoli CA, Selinger DA, Pikaard CS, Richards EJ, et al. Analysis of histone acetyltransferase and histone deacetylase families of Arabidopsis thaliana suggests functional diversification of chromatin modification among multicellular eukaryotes. Nucleic acids Res. 2002; 30:5036-5055.

8. Ekwall K. Genome-wide analysis of HDAC function. Trends Genet. 2005; 21:608-615.

9. Wang Z, Cao H, Chen F, Liu Y. The roles of histone acetylation in seed performance and plant development. Plant Physiol Bioch. 2014; 84:125-133.

10. Zhao T, Zhan Z, Jiang D. Histone modifications and their regulatory roles in plant development and environmental memory. J Genet Genomics. 2019; 46:467-476.

11. Jiang J, Ding AB, Liu F, Zhong X. Linking signaling pathways to histone acetylation dynamics in plants. J Exp Bot. 2020; 71: 5179-5190.

12. Ueda M, Seki M. Histone modifications form epigenetic regulatory networks to regulate abiotic stress response. Plant Physiol. 2020; 182: 15-26.

13. Cohen R, Schocken J, Kaldis A, Vlachonasios KE, Hark AT, McCain ER. The histone acetyltransferase GCN5 affects the inflorescence meristem and stamen development in Arabidopsis. Planta. 2009; 230:1207-1221.

14. Hu Z, Song N, Zheng M, Liu X, Liu Z. Xing J, et al. Histone acetyltransferase GCN 5 is essential for heat stress-responsive gene activation and thermotolerance in Arabidopsis. Plant J. 2015; 84:1178- 
1191.

15. Li S, Lin Y, Wang P, Zhang B, Li M, Chen S, et al. The AREB1 transcription factor influences histone acetylation to regulate drought responses and tolerance in Populus trichocarpa. Plant Cell. 2019; $31: 663-686$.

16. Zheng M, Liu X, Lin J, Liu X, Wang Z, Xin M, et al. Histone acetyltransferase GCN 5 contributes to cell wall integrity and salt stress tolerance by altering the expression of cellulose synthesis genes. Plant J. 2019; 97:587-602.

17. Xiao J, Zhang H, Xing L, Xu S, Liu H, Chong K, Xu Y. Requirement of histone acetyltransferases HAM1 and HAM2 for epigenetic modification of FLC in regulating flowering in Arabidopsis. J Plant Physiol. 2013; 170:444-451.

18. van Zanten M, Zöll C, Wang Z, Philipp C, Carles A, Li Y, et al. HISTONE DEACETYLASE 9 represses seedling traits in Arabidopsis thaliana dry seeds. Plant J. 2014; 80:475-488.

19. Zheng Y, Ding Y, Sun X, Xie S, Wang D, Liu X, Su L, Lei Pan W, Zhou DX. Histone deacetylase HDA9 negatively regulates salt and drought stress responsiveness in Arabidopsis. J Exp Bot. 2016; 67:1703-1713.

20. Chen LT, Wu K. Role of histone deacetylases HDA6 and HDA19 in ABA and abiotic stress response. Plant Signal Behav. 2010; 5: 1318-

21. Mehdi S, Derkacheva M, Ramström M, Kralemann L, Bergquist J, Hennig L. The WD40 domain protein MSI1 functions in a histone deacetylase complex to fine-tune abscisic acid signaling. Plant Cell. 2016; 28:42-54.

22. Ueda M, Matsui A, Tanaka M, Nakamura T, Abe T, Sako K, et al. The distinct roles of class I and II RPD3-like histone deacetylases in salinity stress response. Plant Physiol. 2017; 175:1760-1773.

23. Ueda M, Matsui A, Nakamura T, Abe T, Sunaoshi Y, Shimada H, Seki M. Versatility of HDA19deficiency in increasing the tolerance of Arabidopsis to different environmental stresses. Plant Signal Behav. 2018; 13:e1475808.

24. Zhao J, Li M, Gu D, Liu X, Zhang J, Wu K, Zhang X, Teixeira da Silva J, Duan J. Involvement of rice histone deacetylase HDA705 in seed germination and in response to ABA and abiotic stresses. Biochem Bioph Res Co. 2016; 470:439-444.

25. Liu X, Luo M, Zhang W, Zhao J, Zhang J, Wu K, et al. Histone acetyltransferases in rice (Oryza sativa): phylogenetic analysis, subcellular localization and expression. BMC Plant Biol. 2012; $12: 145$.

26. Tan S, Gao L, Li T, Chen L. Phylogenetic and expression analysis of histone acetyltransferases in Brachypodium distachyon. Genomics. 2019; 111:1966-1976.

27. Peng M, Ying P, Liu X, Li C, Xia R, Li J, Zhao M. Genome-wide identification of histone modifiers and their expression patterns during fruit abscission in Litchi. Front Plant Sci. 2017; 8:639.

28. Aquea F, Timmermann T, Arce-Johnson P. Analysis of histone acetyltransferase and deacetylase families of Vitis vinifera. Plant Physiol Bioch. 2010; 48:194-199. 
29. Xu J, Xu H, Liu Y, Wang X, Xu Q, Deng X. Genome-wide identification of sweet orange (Citrus sinensis) histone modification gene families and their expression analysis during the fruit development and fruit-blue mold infection process. Front Plant Sci. 2015; 6:607.

30. Imran M, Shafiq S, Farooq MA, Naeem MK, Widemann E, Bakhsh A, et al. Comparative genome-wide analysis and expression profiling of histone acetyltransferase (HAT) gene family in response to hormonal applications, metal and abiotic stresses in cotton. Int J Mol Sci. 2019; 20:5311.

31. Hollender C, Liu Z. Histone deacetylase genes in ArabidopsisJ Integr Plant Biol. 2008; 50:875-885.

32. Hu Y, Qin F, Huang L, Sun Q, Li C, Zhao Y, Zhou DX. Rice histone deacetylase genes display specific expression patterns and developmental functions. Biochem Bioph Res Co. 2009; 388:266-271.

33. Liew LC, Singh MB, Bhalla PL. An RNA-seq transcriptome analysis of histone modifiers and RNA silencing genes in soybean during floral initiation process. PLoS One. 2013; 8:e77502.

34. Zhao L, Lu J, Zhang J, Wu PY, Yang S, Wu K. Identification and characterization of histone deacetylases in tomato (Solanum lycopersicum). Front Plant Sci. 2015; 5:760.

35. Imran M, Shafiq S, Naeem MK, Widemann E, Munir MZ, Jensen KB, Wang R. Histone Deacetylase (HDAC) Gene Family in Allotetraploid Cotton and Its Diploid Progenitors: In Silico Identification, Molecular Characterization, and Gene Expression Analysis under Multiple Abiotic Stresses, DNA Damage and Phytohormone Treatments. Int J Mol Sci. 2020; 21:321.

36. Ramírez-González RH, Borrill P, Lang D, Harrington SA, Brinton J, Venturini L, et al. The transcriptional landscape of polyploid wheat. Science. 2018; 361:

37. Bienz M. The PHD finger, a nuclear protein-interaction domain. Trends Biochem. Sci. 2006; 31:35-40.

38. Gamsjaeger R, Liew CK, Loughlin FE, Crossley M, Mackay JP. Sticky fingers: Zinc-fingers as proteinrecognition motifs. Trends Biochem Sci. 2007; 32:63-70.

39. Marmorstein R, Berger SL. Structure and function of bromodomains in chromatin-regulating complexes. Gene. 2001; 272:1-9.

40. Servet C, e Silva NC, Zhou DX. Histone acetyltransferase AtGCN5/HAG1 is a versatile regulator of developmental and inducible gene expression in Arabidopsis. Mol Plant. 2010; 3:670-677.

41. Wu X, Oh MH, Schwarz EM, Larue CT, Sivaguru M, Imai BS, et al. Lysine acetylation is a widespread protein modification for diverse proteins in Arabidopsis. Plant Physiol. 2011; 155:1769-

42. Nallamilli BR, Edelmann MJ, Zhong X, Tan F, Mujahid H, Zhang J, Nanduri B, Peng Z. Global analysis of lysine acetylation suggests the involvement of protein acetylation in diverse biological processes in rice (Oryza sativa). PLoS One. 2014; 9:e89283.

43. Xue C, Liu S, Chen C, Zhu J, Yang X, Zhou Y, et al. Global proteome analysis links lysine acetylation to diverse functions in Oryza sativa. Proteomics. 2018; 18: 1700036.

44. Ma X, Zhang B, Liu C, Tong B, Guan T, Xia D. Expression of a populus histone deacetylase gene 84KHDA903 in tobacco enhances drought tolerance. Plant Sci. 2017; 265:1-

45. Yu X, Gao Q, Chen G, Guo JE, Guo X, Tang B, Hu Z. SIHDA5, a tomato histone deacetylase gene, is involved in responding to salt, drought, and ABA. Plant Mol Biol Rep. 2018; 36:36- 
46. Song J, Henry HA, Tian L. Brachypodium histone deacetylase BdHD1 positively regulates ABA and drought stress responses. Plant Sci. 2019; 283:355-

47. Zhang JB, He SP, Luo JW, Wang XP, Li DD, Li XB. A histone deacetylase, GhHDT4D, is positively involved in cotton response to drought stress. Plant Mol Biol. 2020; 104: 67-

48. Tamura K, Stecher G, Peterson D, Filipski A, Kumar S. MEGA6: molecular evolutionary genetics analysis version 6.0. Mol Biol Evol. 2013; 30:2725-2729.

49. Chen $\mathrm{C}$, Chen $\mathrm{H}$, Zhang $\mathrm{Y}$, Thomas HR, Frank MH, HeY, Xia R. TBtools-an integrative toolkit developed for interactive analyses of big biological data. Mol Plant. 2020; 13:1194-1202.

50. Garrido J, Aguilar M, Prieto P. Identification and validation of reference genes for RT-qPCR normalization in wheat meiosis. Sci Rep. 2020; 10:1-12.

\section{Figures}



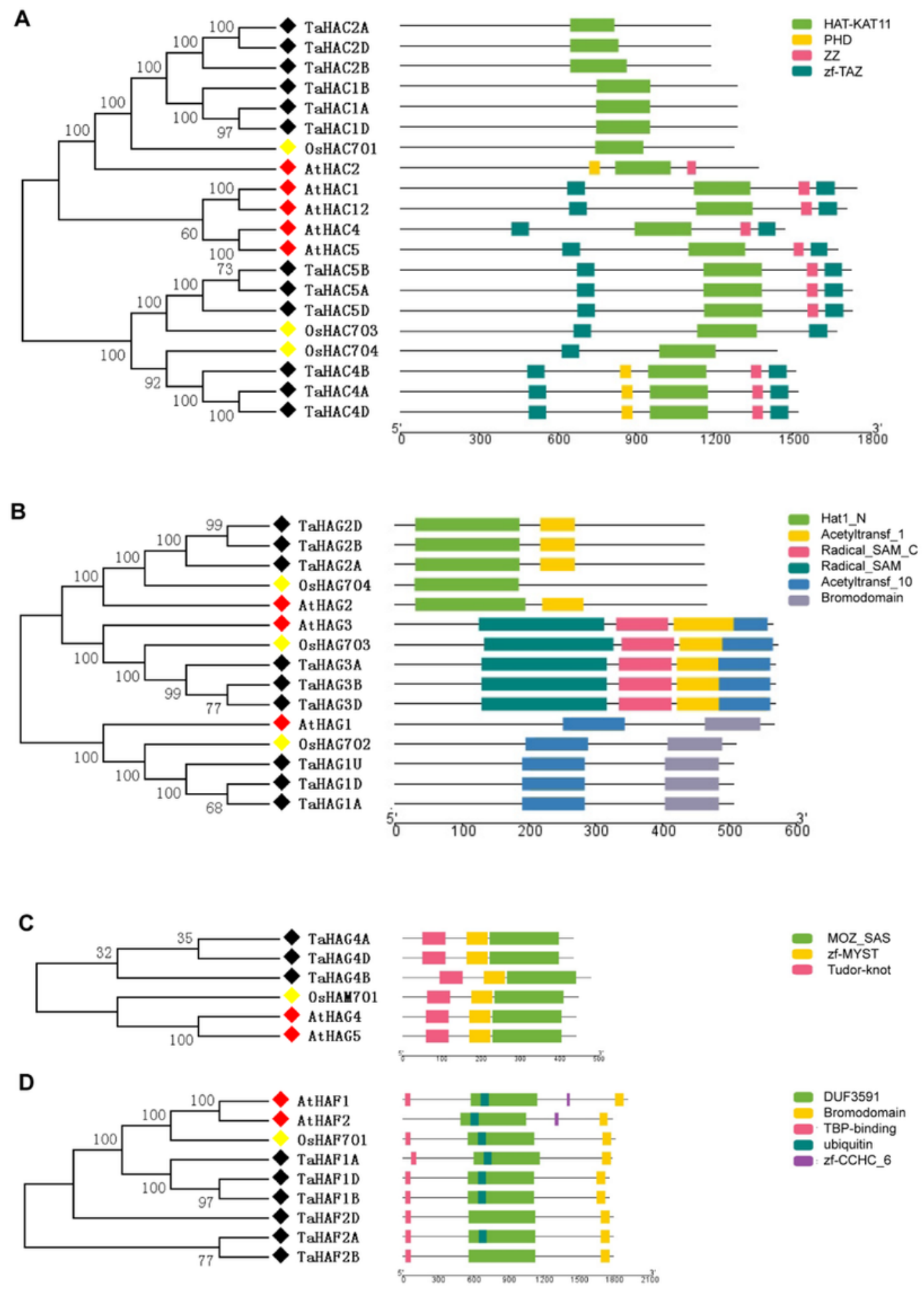

\section{Figure 1}

Molecular phylogenetic analysis and domain structures of HATs in Triticum aestivum, Arabidopsis thaliana and Oryza sativa. (A) CBP subfamily. (B) GNAT subfamily. (C) MYST subfamily and (D) TAFII250 subfamily. The HATs from Triticum aestivum, Oryza sativa, and Arabidopsis thaliana are marked with black, yellow, and red diamonds, respectively. 

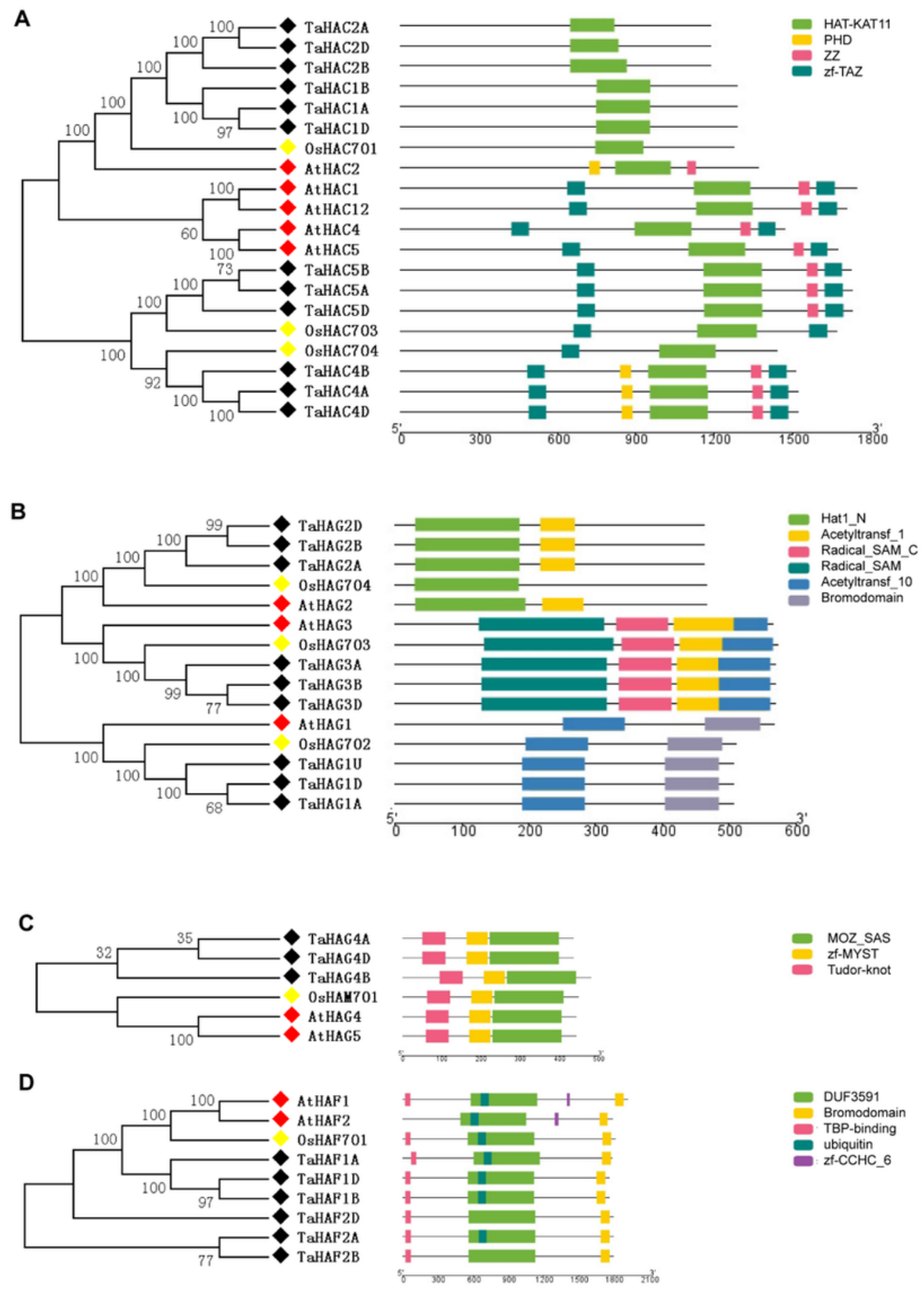

\section{Figure 1}

Molecular phylogenetic analysis and domain structures of HATs in Triticum aestivum, Arabidopsis thaliana and Oryza sativa. (A) CBP subfamily. (B) GNAT subfamily. (C) MYST subfamily and (D) TAFII250 subfamily. The HATs from Triticum aestivum, Oryza sativa, and Arabidopsis thaliana are marked with black, yellow, and red diamonds, respectively. 
A

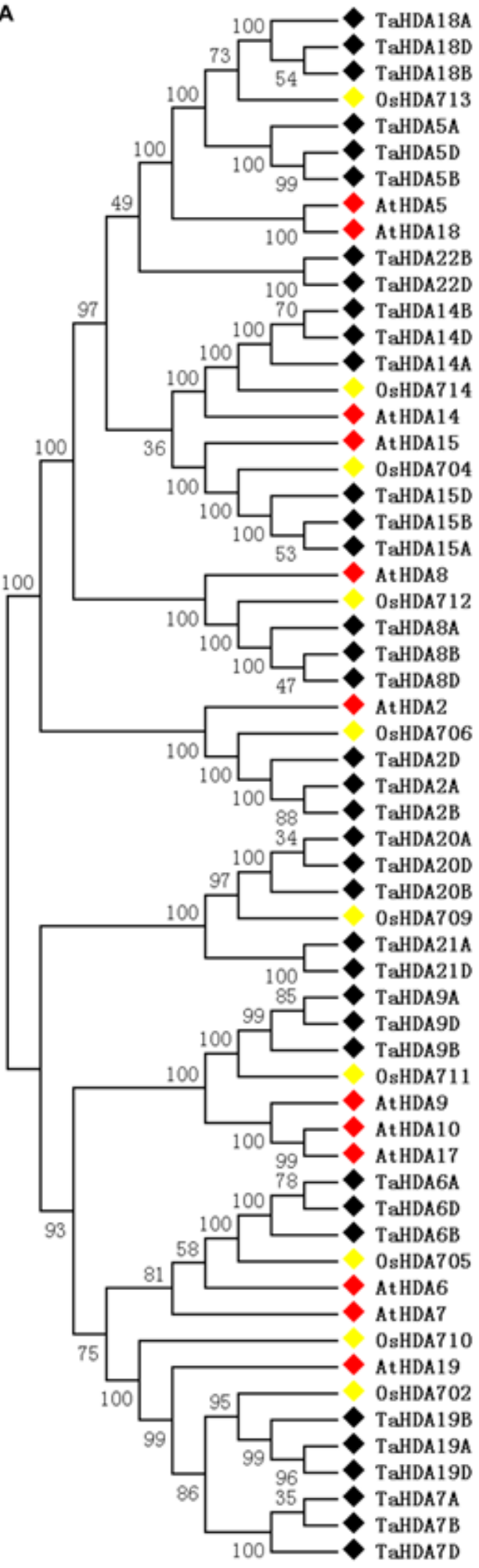

B

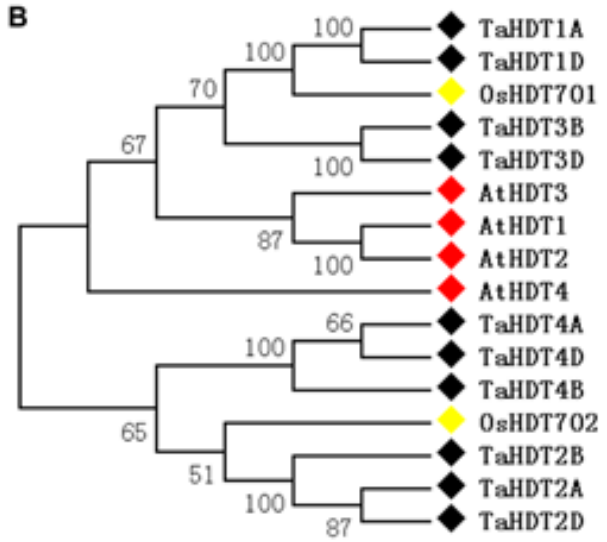

c

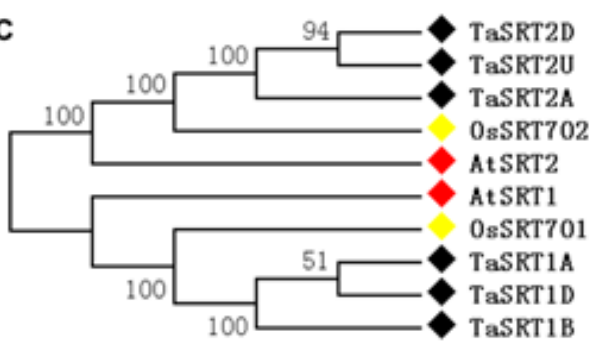

D
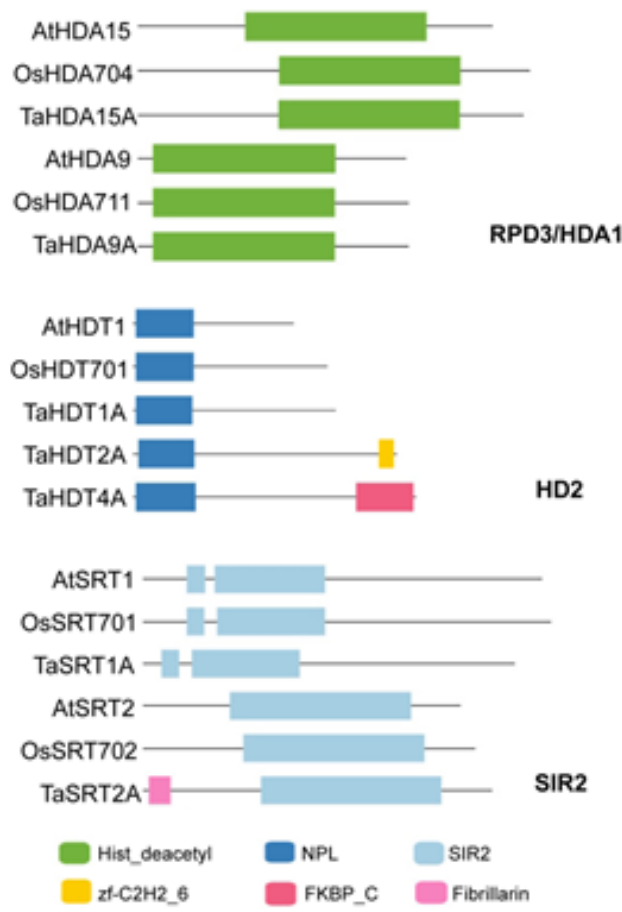

Figure 2

Molecular phylogenetic analysis and domain structures of HDACs in Triticum aestivum, Arabidopsis thaliana, and Oryza sativa. (A) RPD3/HDA1 subfamily. (B) HD2 subfamily. (C) SIR2 subfamily and (D) Typical domains of HDAC genes in each subfamily. The HDACs from Triticum aestivum, Oryza sativa, and Arabidopsis thaliana are marked with black, yellow, and red diamonds, respectively. 
A

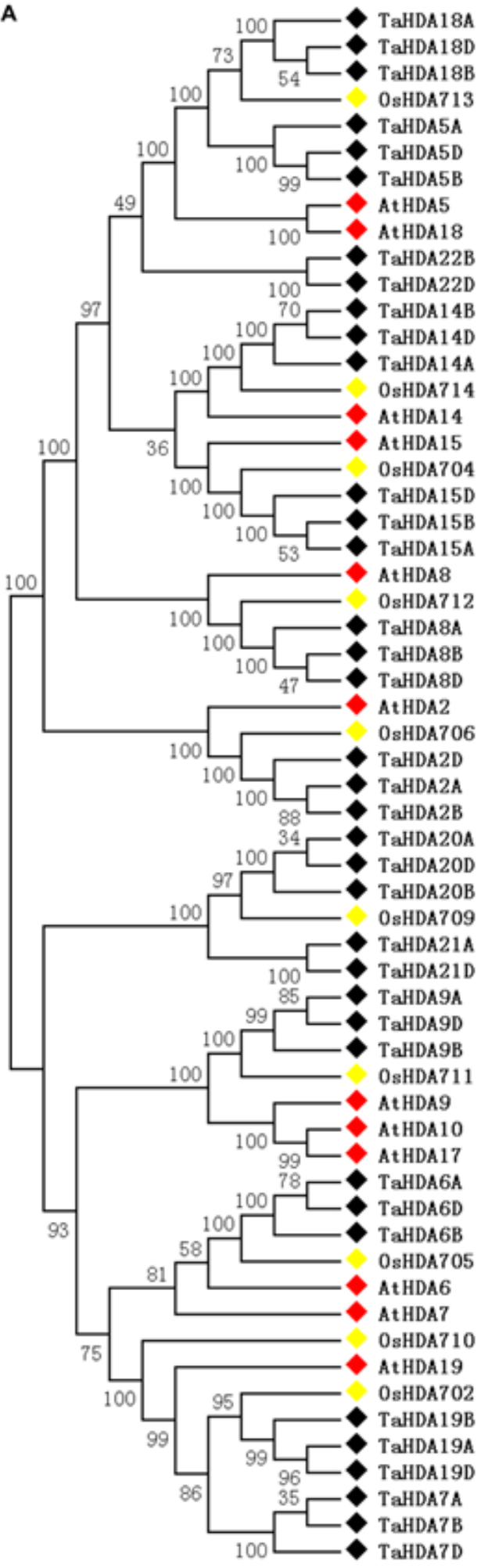

B
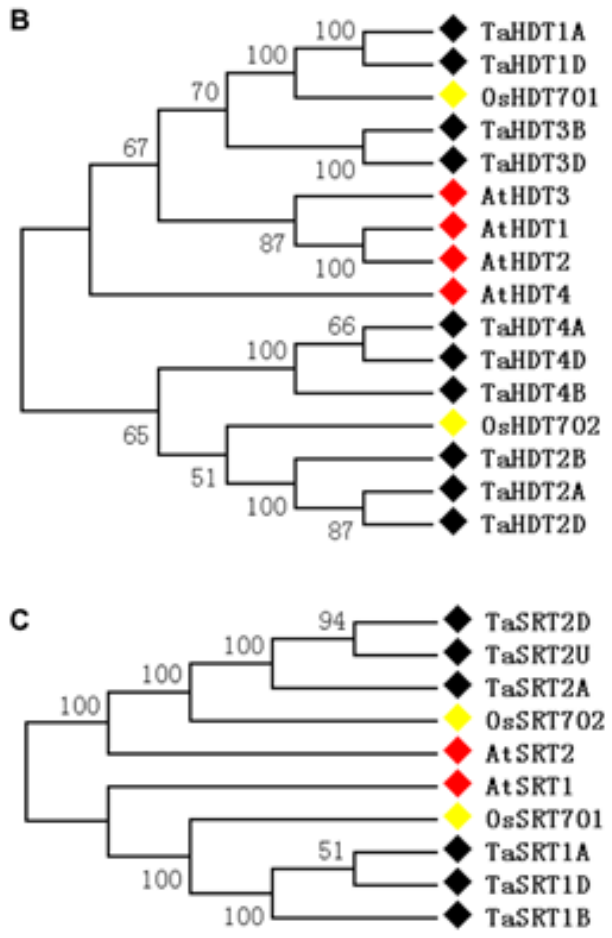

D
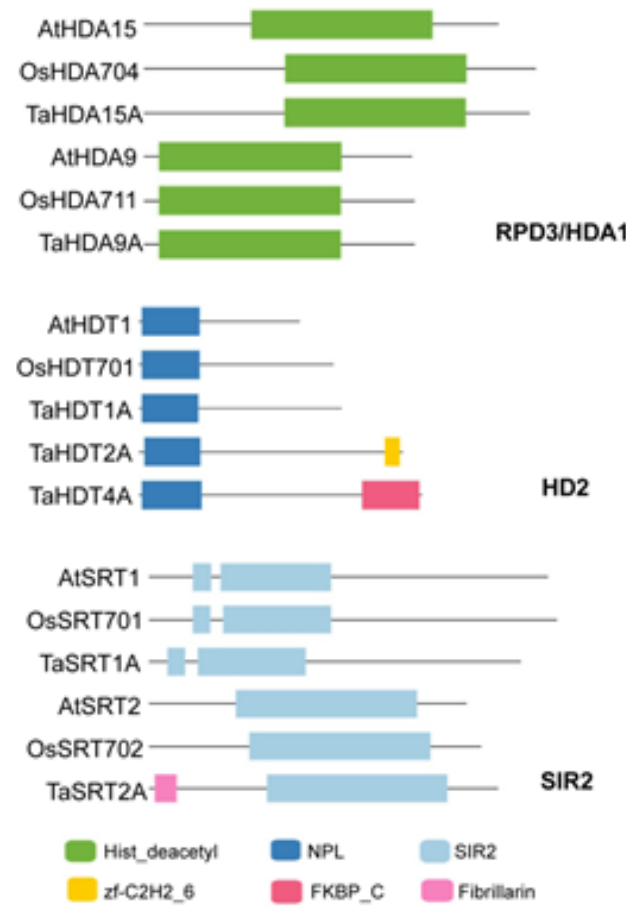

Figure 2

Molecular phylogenetic analysis and domain structures of HDACs in Triticum aestivum, Arabidopsis thaliana, and Oryza sativa. (A) RPD3/HDA1 subfamily. (B) HD2 subfamily. (C) SIR2 subfamily and (D) Typical domains of HDAC genes in each subfamily. The HDACs from Triticum aestivum, Oryza sativa, and Arabidopsis thaliana are marked with black, yellow, and red diamonds, respectively. 

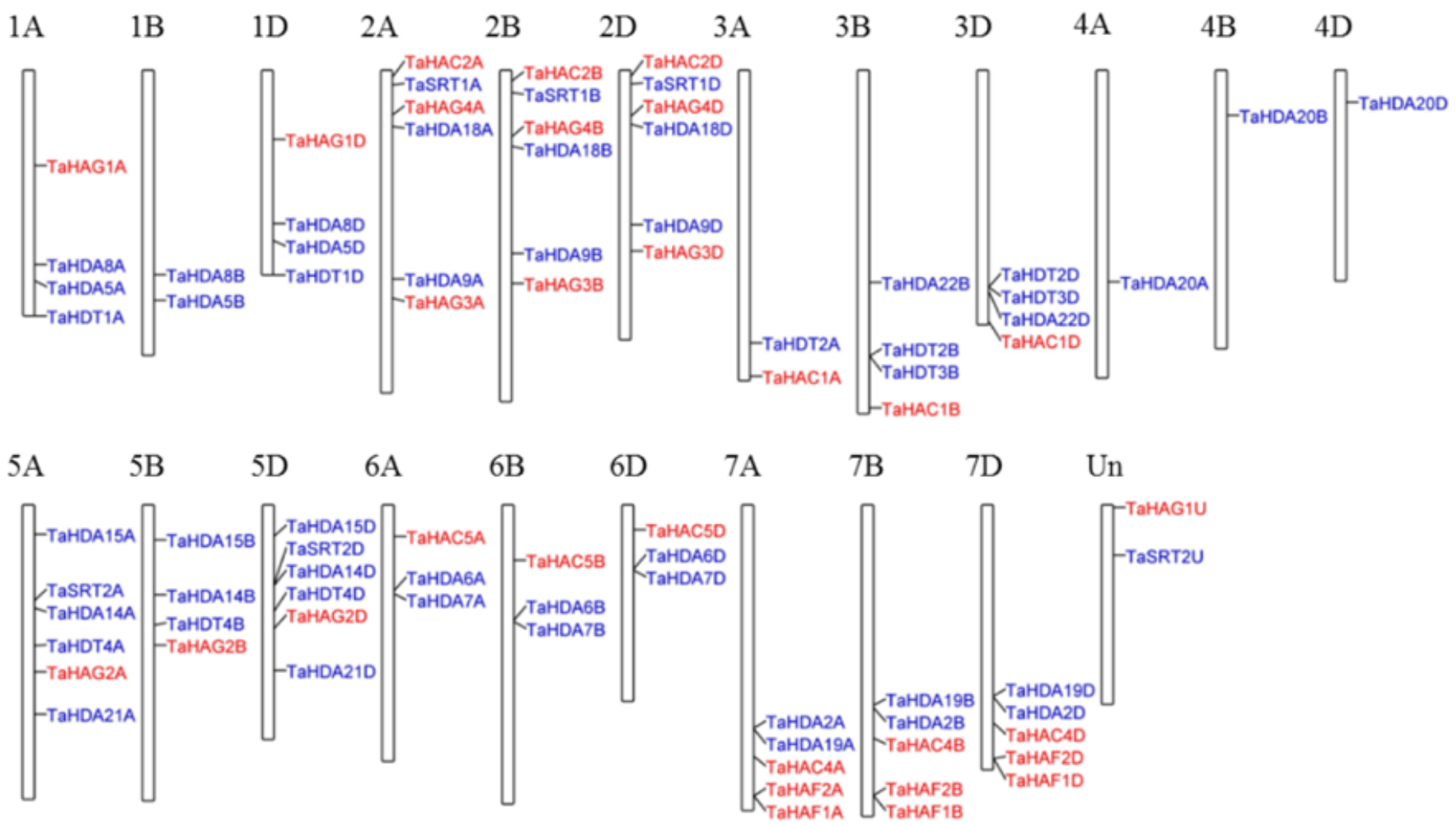

\section{Figure 3}

Chromosomes locations of TaHATs and TaHDACs genes on the wheat genome. TaHATs and TaHDACs are marked with red and blue, respectively. 

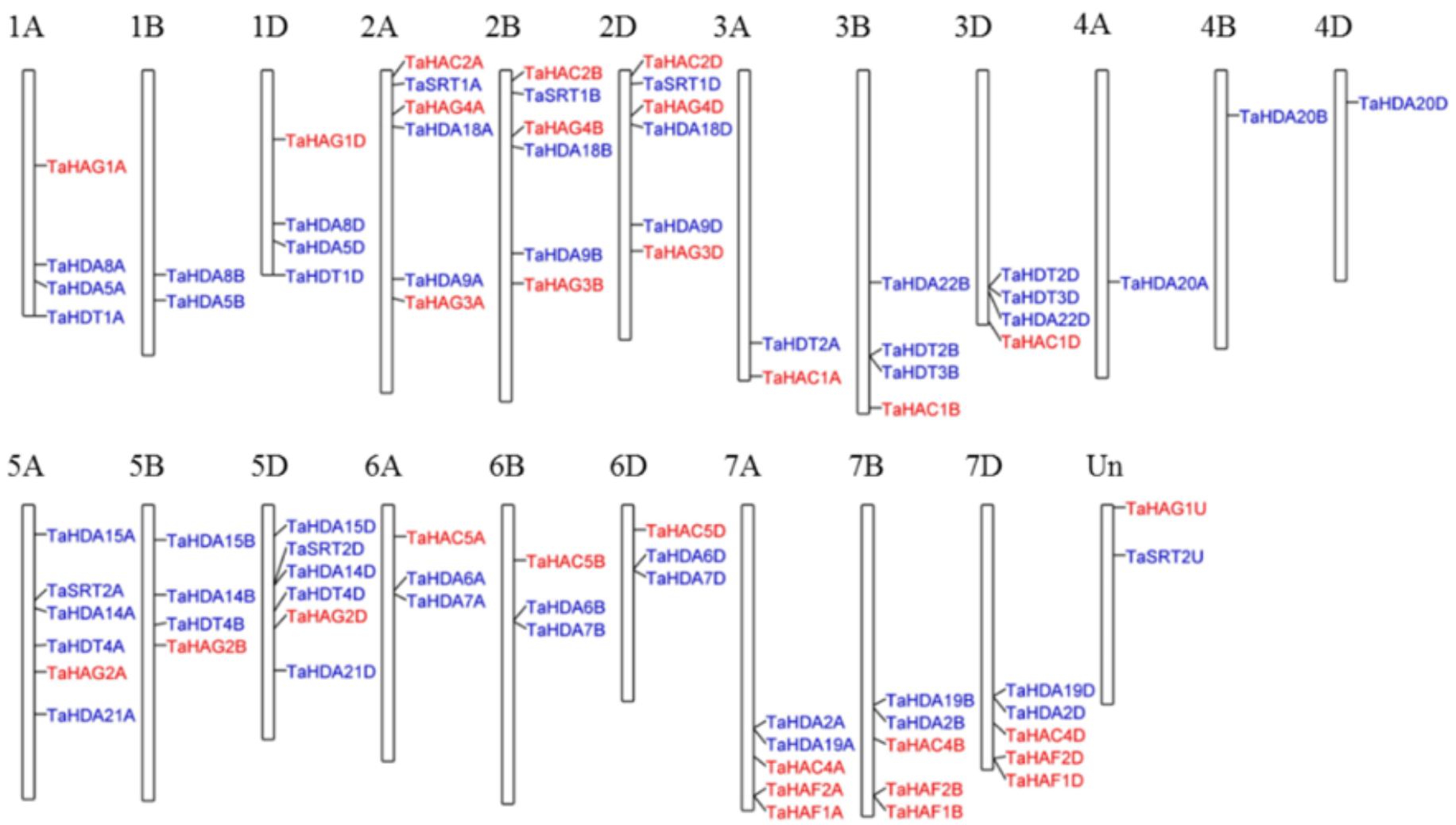

\section{Figure 3}

Chromosomes locations of TaHATs and TaHDACs genes on the wheat genome. TaHATs and TaHDACs are marked with red and blue, respectively. 
A

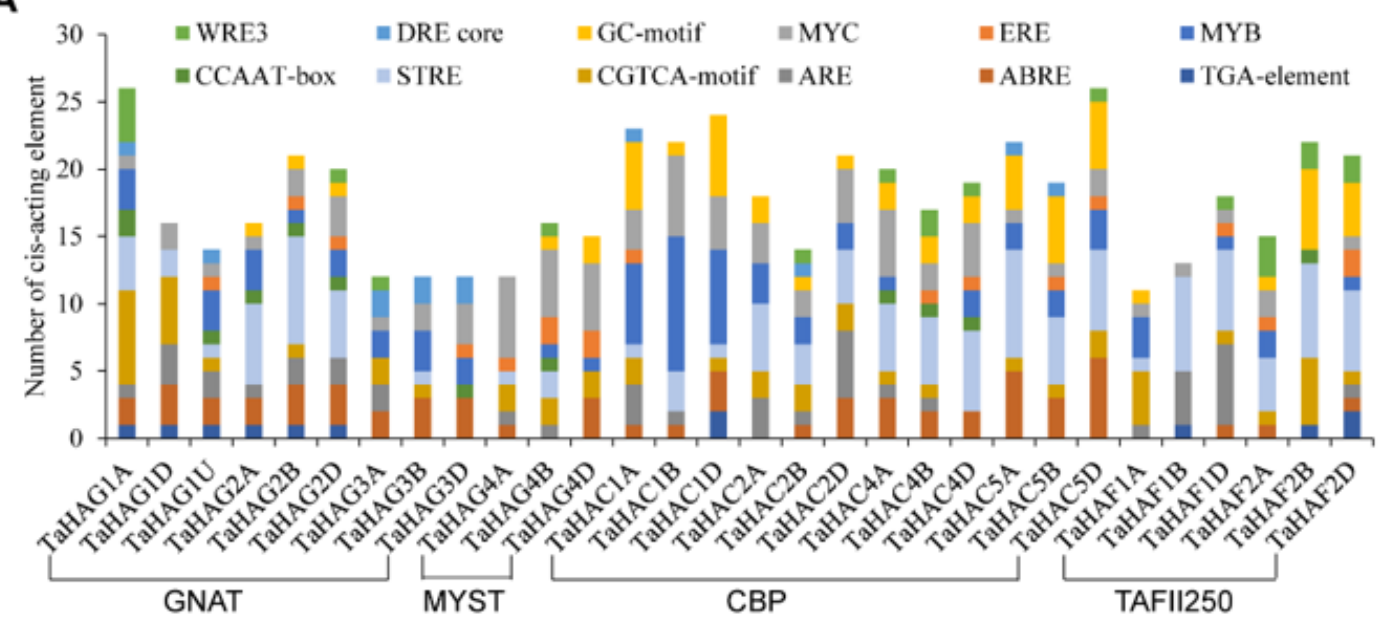

B

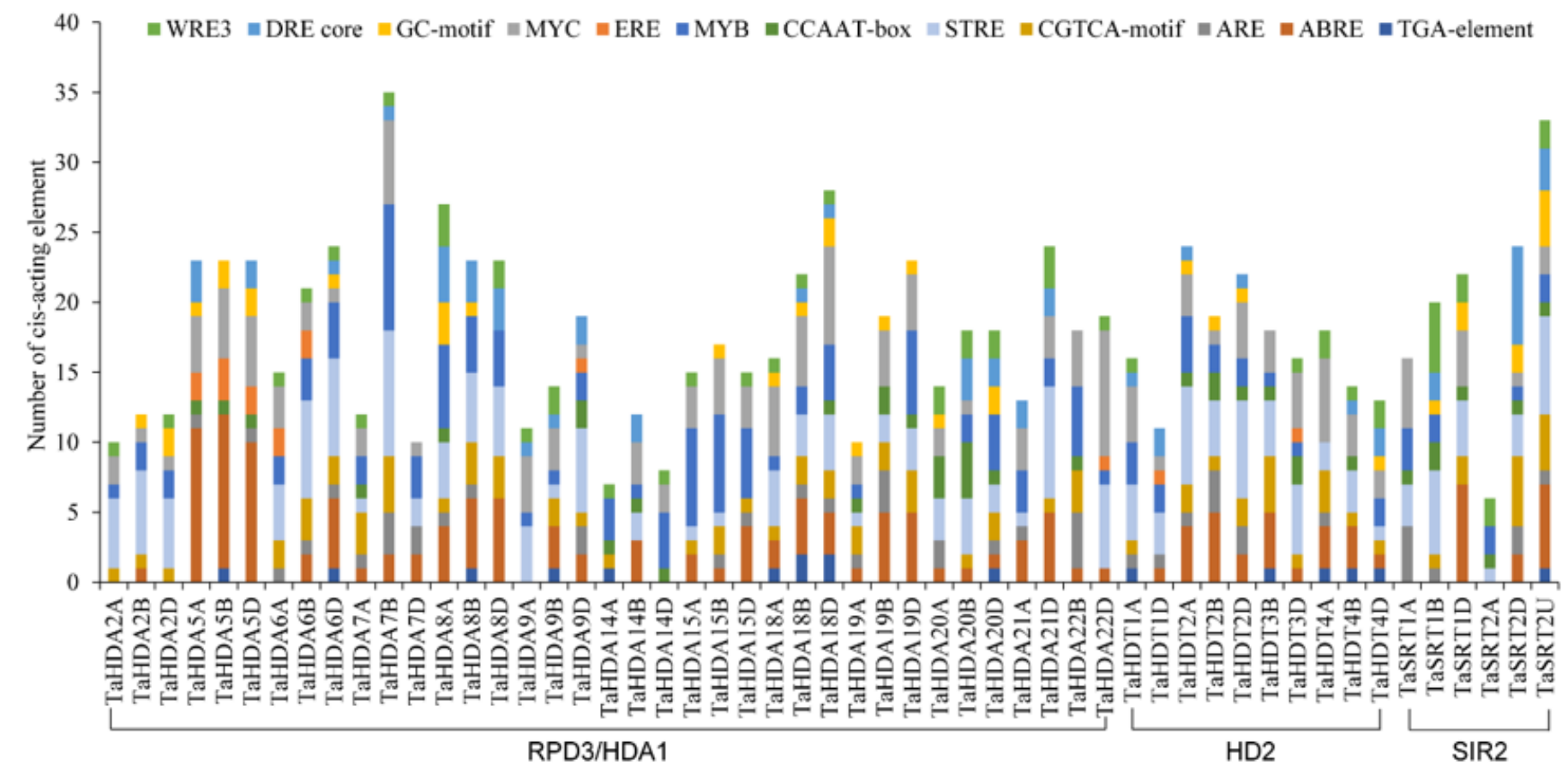

Figure 4

The number of putative cis-regulatory elements in the HATs (A) and HDACs (B) promoters. 
A

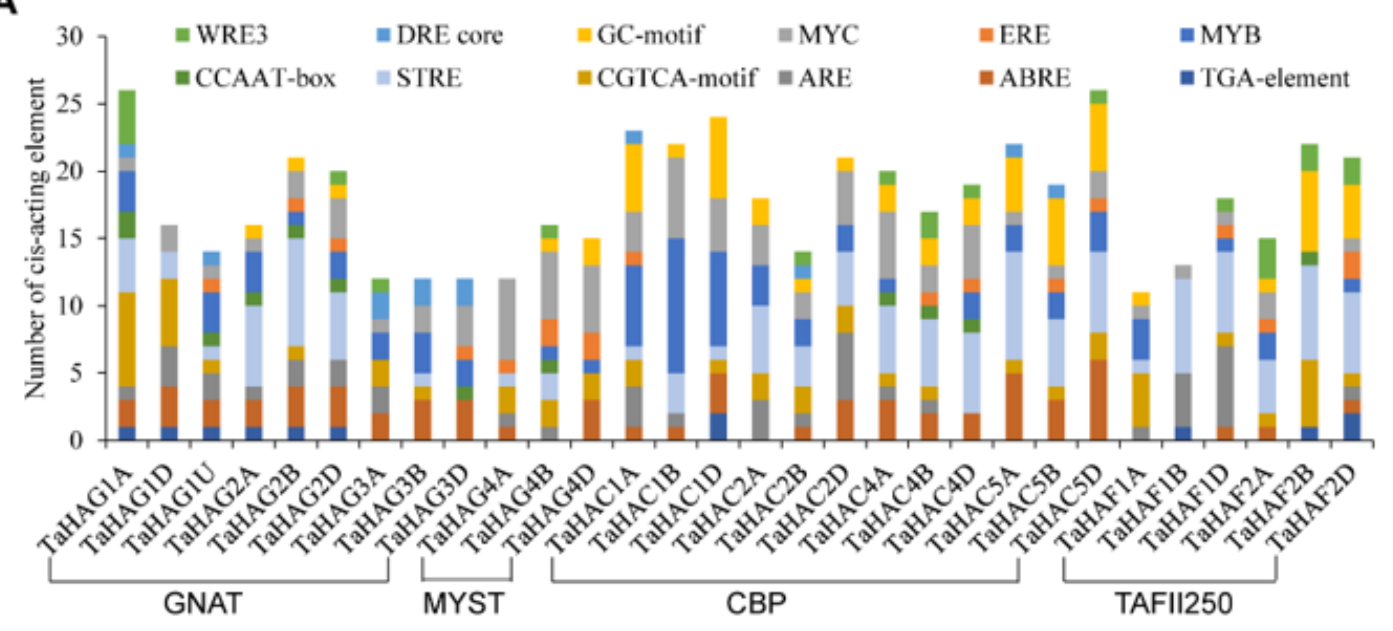

B

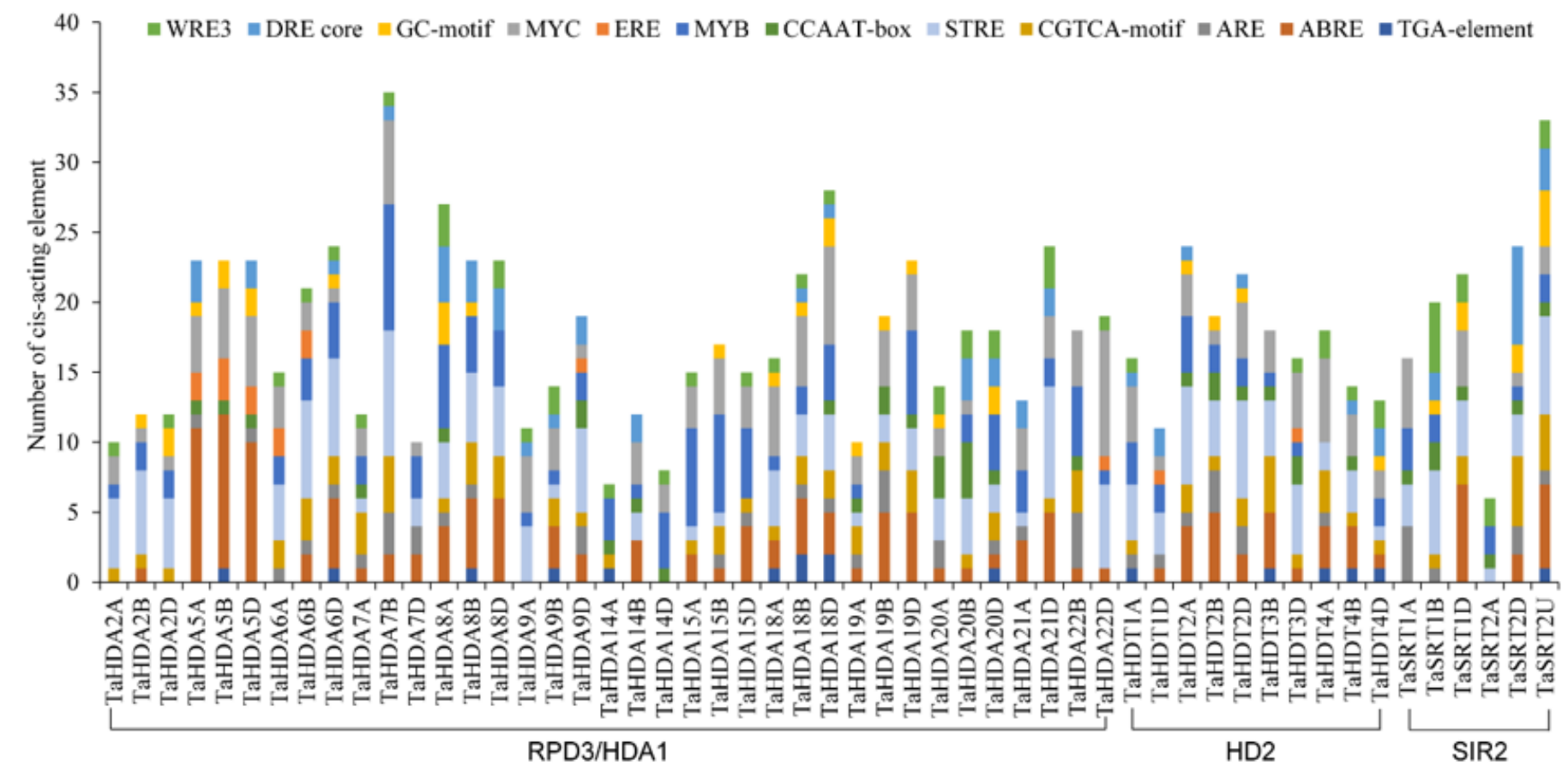

Figure 4

The number of putative cis-regulatory elements in the HATs (A) and HDACs (B) promoters. 
A

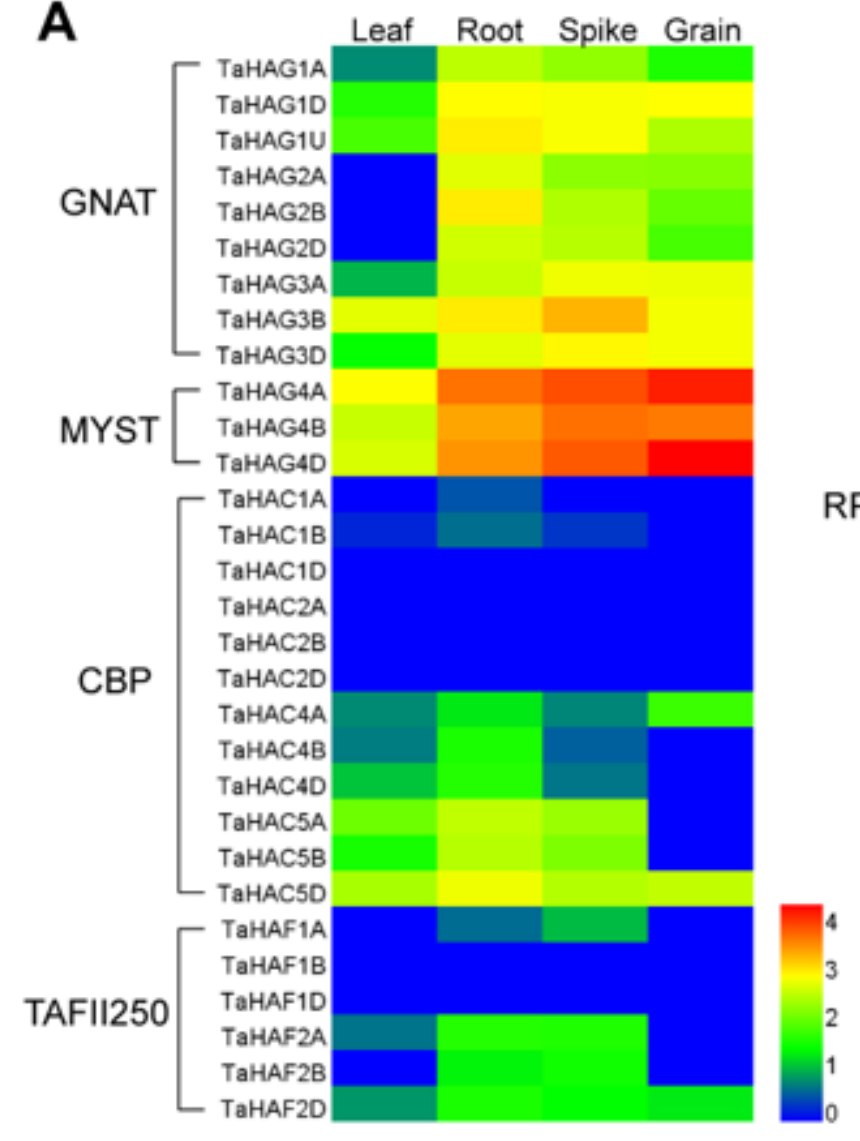

B

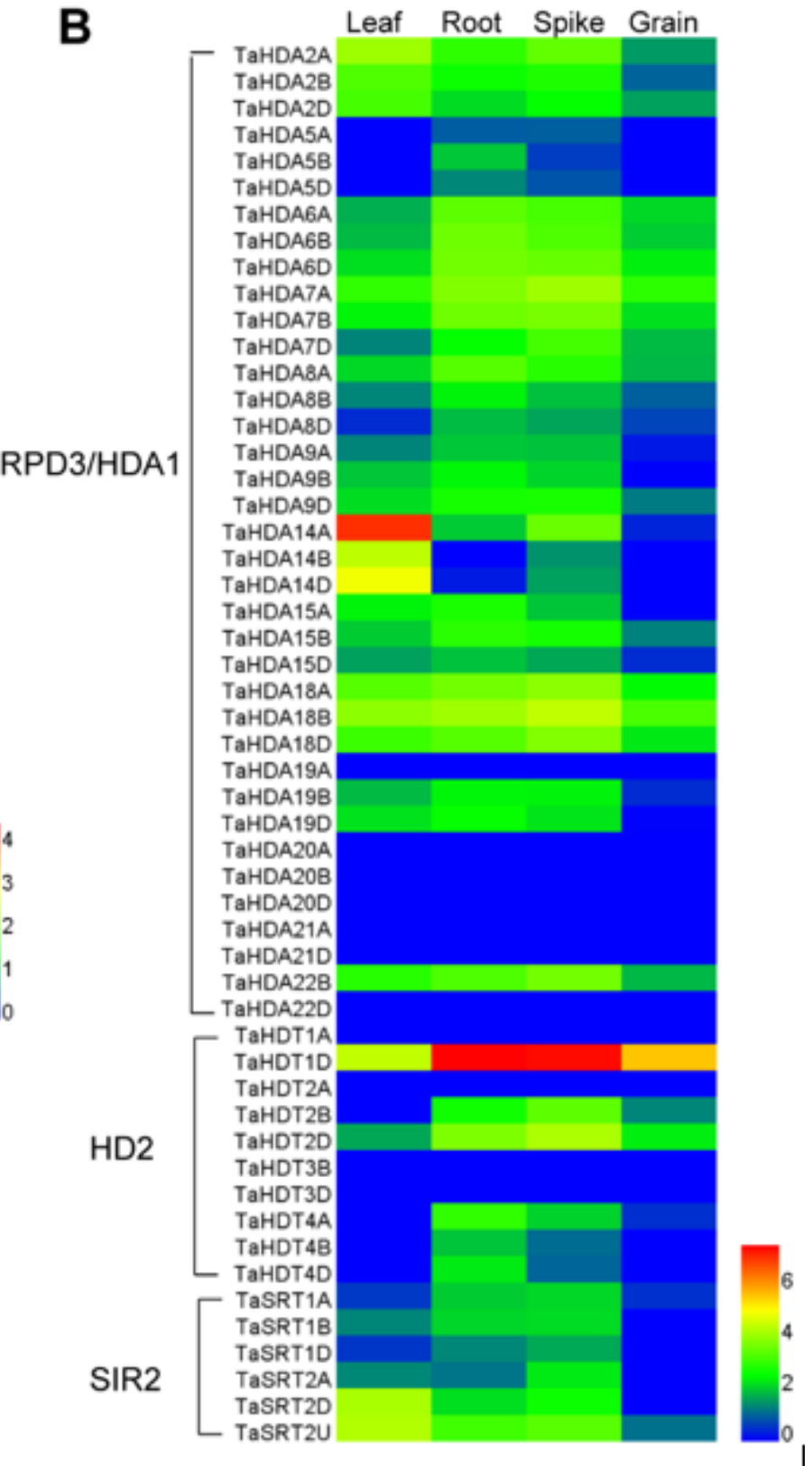

\section{Figure 5}

Gene expression pattern of wheat HATs (A) and HDACs (B) in different tissues. The expression levels (TPM values) of TaHATs and TaHDACs in the leaf, root, spike, and grain were downloaded from expVIP. 
A

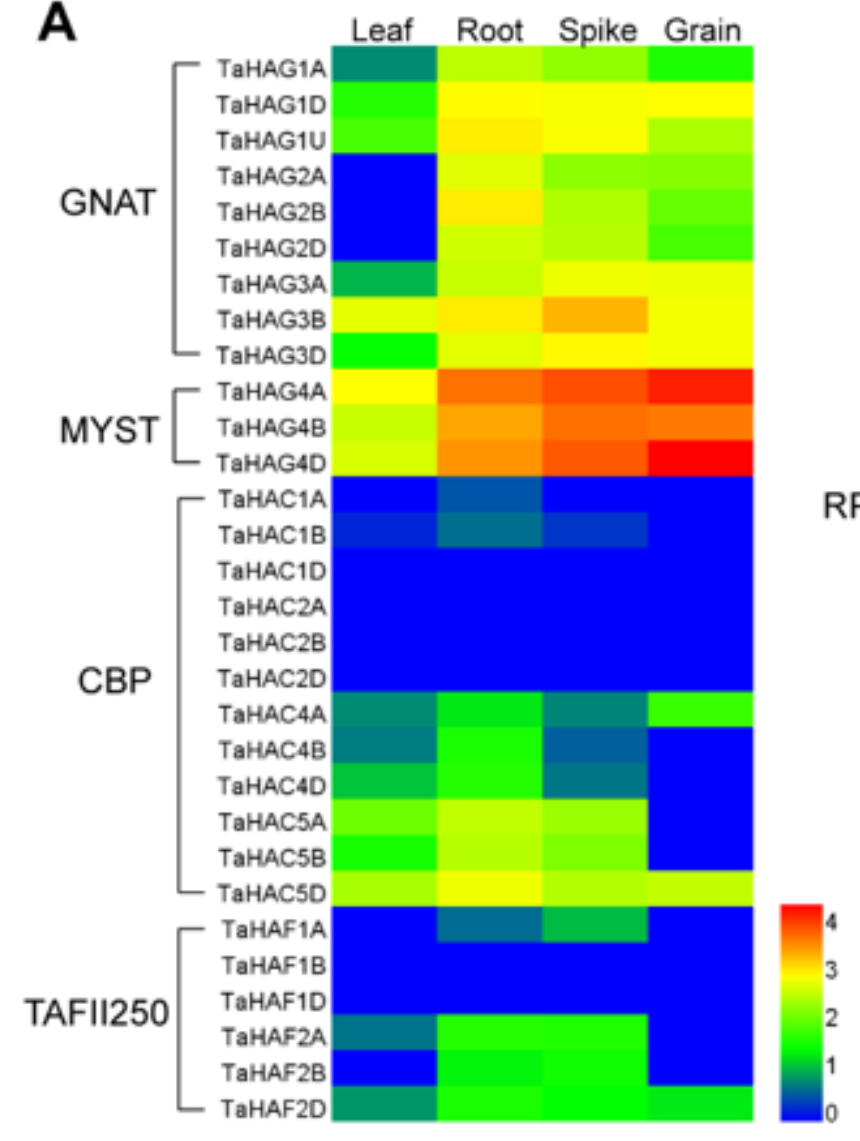

B

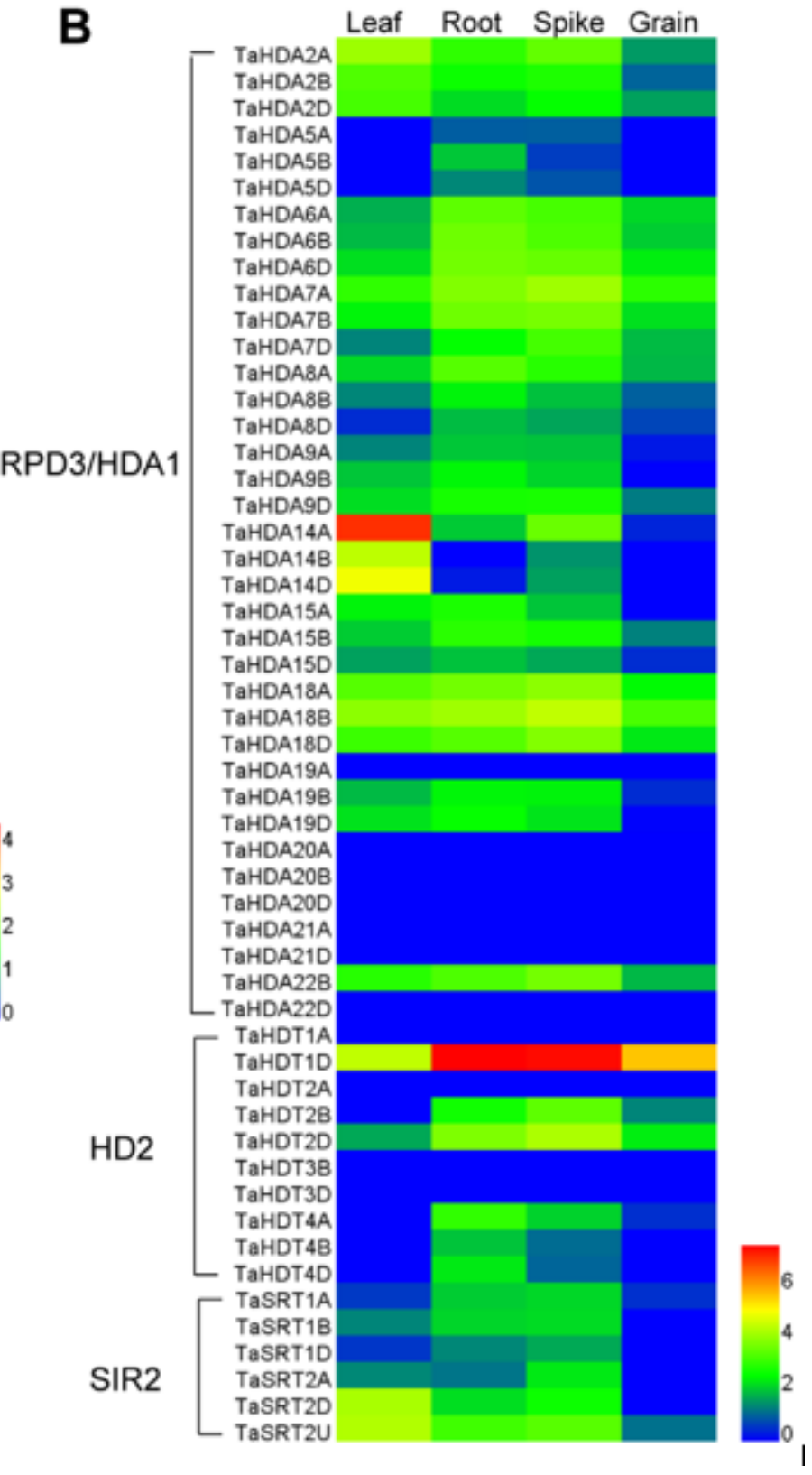

\section{Figure 5}

Gene expression pattern of wheat HATs (A) and HDACs (B) in different tissues. The expression levels (TPM values) of TaHATs and TaHDACs in the leaf, root, spike, and grain were downloaded from expVIP. 
A

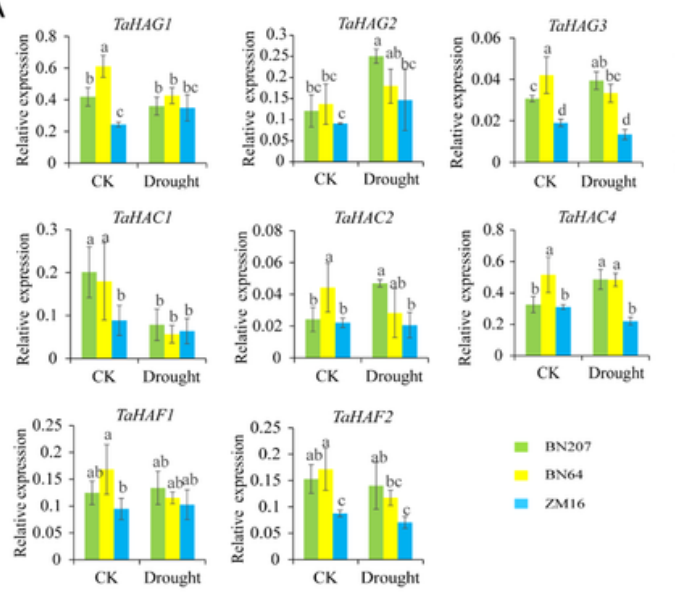

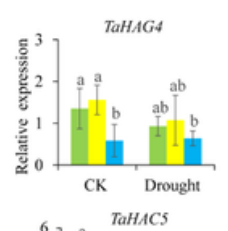
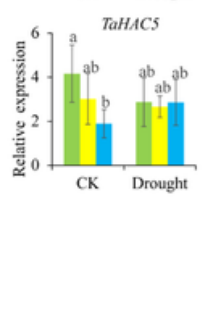

B
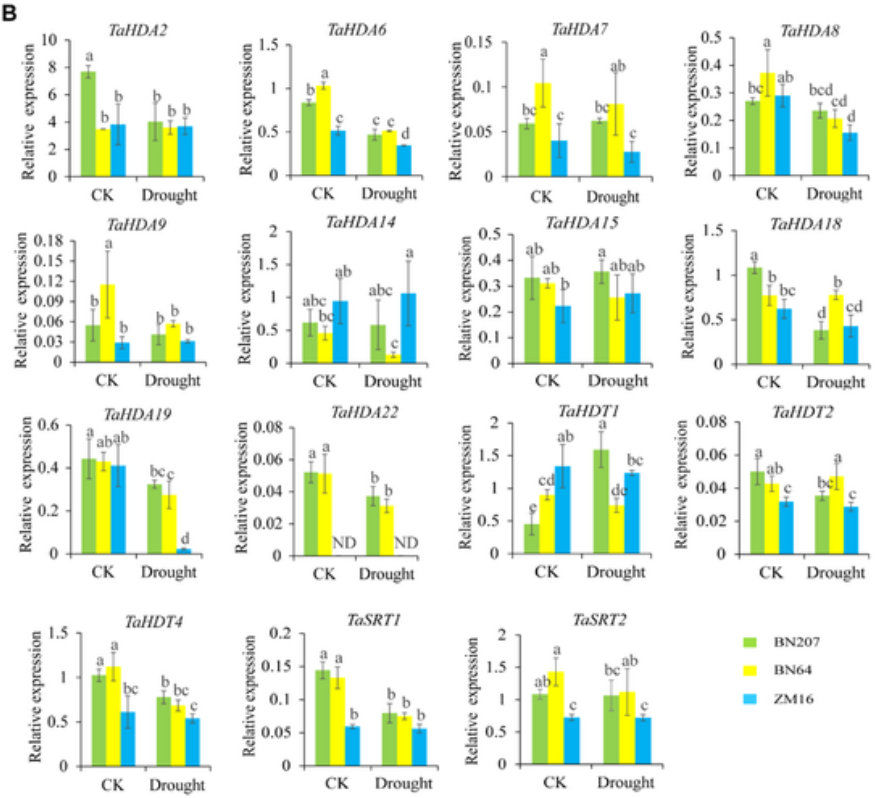

Figure 6

qRT-PCR analysis of the expression of TaHATs and TaHDACs gene under drought stress. At the booting stage of wheat, control soil water content to $55 \%$ as drought treatment. After 10 days later, flag leaves were collected for the gene expression analysis.

A

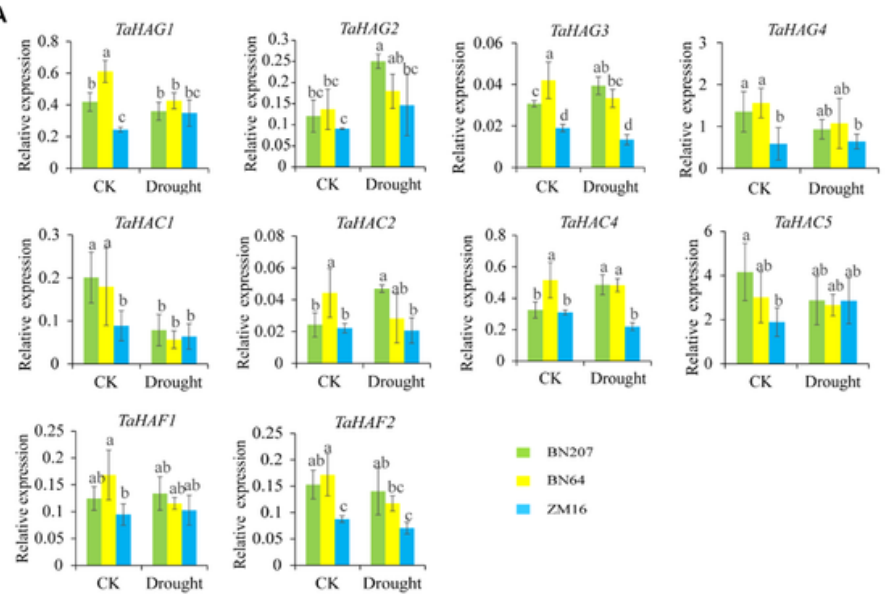

B
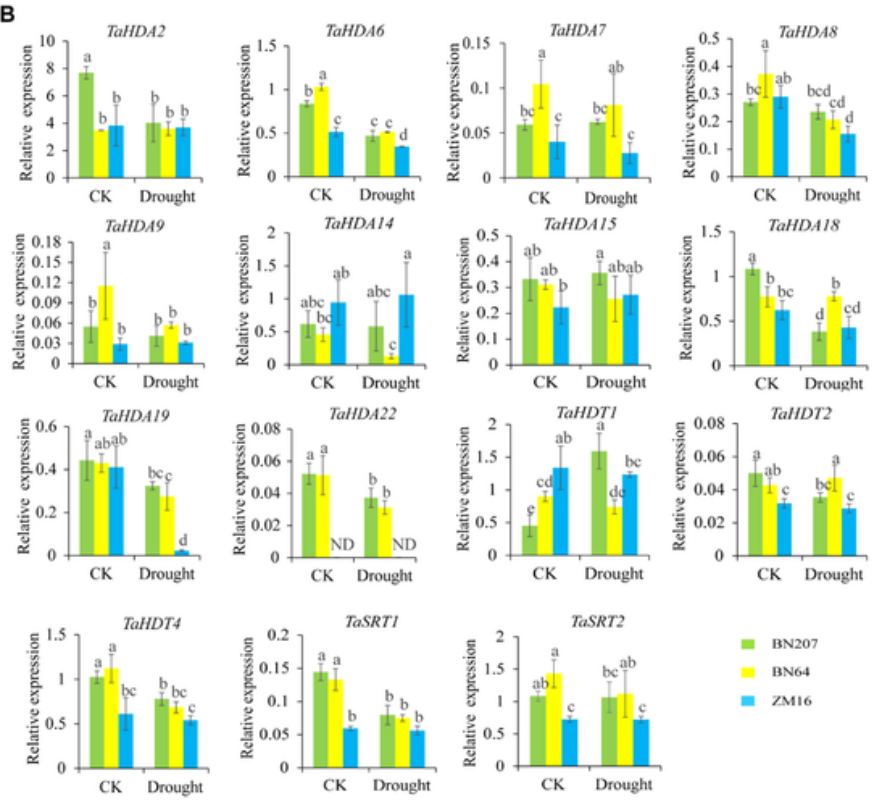

= $\mathrm{BN} 207$

11: BN64

\section{Figure 6}

qRT-PCR analysis of the expression of TaHATs and TaHDACs gene under drought stress. At the booting stage of wheat, control soil water content to $55 \%$ as drought treatment. After 10 days later, flag leaves were collected for the gene expression analysis. 


\section{Supplementary Files}

This is a list of supplementary files associated with this preprint. Click to download.

- Figures1.docx

- FigureS1.docx

- TableS1.xIsx

- TableS1.xlsx

- Tables2.xIsx

- TableS2.xIsx

- TableS3.xIsx

- TableS3.xIsx

- TableS4.docx

- TableS4.docx

- TableS5.xlsx

- TableS5.xIsx 